\title{
Full-Duplex Relay Selection in Cognitive Underlay Networks
}

\author{
Mohammad Galal Khafagy, Senior Member, IEEE, Mohamed-Slim Alouini, Fellow, IEEE, \\ and Sonia Aïssa, Senior Member, IEEE
}

\begin{abstract}
We analyze the outage and throughput performance of full-duplex relay selection (FDRS) in underlay cognitive networks. Contrary to half-duplex relaying, full-duplex relaying (FDR) enables simultaneous listening/forwarding at the secondary relay(s), thereby allowing for higher spectral efficiency. However, due to simultaneous source/relay transmissions in FDR, the superimposed signal at the primary receiver should now satisfy the existing interference constraint, which can considerably limit the secondary network throughput. In this regard, FDRS can offer an adequate solution to boost the secondary throughput while satisfying the imposed interference limit. We first analyze the performance of opportunistic FDRS with residual selfinterference (RSI) by deriving the exact cumulative distribution function of its end-to-end signal-to-interference-plus-noise ratio under Nakagami- $m$ fading. We also evaluate the offered diversity gain of relay selection for different full-duplex cooperation schemes in the presence/absence of a direct source-destination link under Rayleigh fading. When the RSI link gain model is sublinear in the relay power, which agrees with recent research findings, we show that remarkable diversity can be recovered even in the presence of an interfering direct link. Second, we evaluate the end-to-end performance of FDRS with interference constraints due to the presence of a primary receiver. Finally, the presented theoretical findings are verified by numerical simulations.
\end{abstract}

Keywords-diversity gain, full-duplex, opportunistic relay selection, outage performance, self-interference, spectrum sharing.

\section{INTRODUCTION}

$\mathbf{S}$ PECTRUM sharing cognitive radio represents one paradigm for the celebrated cognitive radio technology in which adequate means are offered to resolve the scarcity problem of wireless resources [1], [2]. Also known as underlay cognitive radio, such a paradigm allows the traffic of secondary cognitive users to coexist with that of the primary spectrum users as long as a certain interference level is not exceeded. In particular, when the secondary source and destination are spatially isolated, relay assistance becomes inevitable to extend the coverage and establish successful communication via relay listening/forwarding, while satisfying the coexistence constraints with the primary user.

The research reported in this publication is supported by funding from King Abdullah University of Science and Technology (KAUST).

This work was done when M. G. Khafagy was with KAUST, Computer, Electrical and Mathematical Science and Engineering Division (CEMSE), Thuwal, Makkah Province, Saudi Arabia (e-mail: mohammad.khafagy@kaust.edu.sa). He is currently affiliated with the Electrical Engineering Department, College of Engineering, Qatar University, Doha, Qatar. M.-S. Alouini is with KAUST, CEMSE, Thuwal, Makkah Province, Saudi Arabia (e-mail: slim.alouini@kaust.edu.sa). S. Aïssa is with the Institut National de la Recherche Scientifique (INRS), University of Quebec, Montreal, QC, H5A 1K6, Canada (e-mail: aissa@emt.inrs.ca).
Cognitive relay networks (CRNs) continue to draw a noticeable interest for their coverage extension capabilities under an efficient spectrum usage [3]. Adhering to the interference constraint in underlay networks, however, can considerably limit the throughput of the secondary system, especially when a single relay is leveraged to assist the secondary sourcedestination communication. To tackle such a challenge, relay selection [4], [5] is adopted in underlay CRNs, offering remarkable performance gains relative to its fixed relaying counterpart. Cognitive relay selection is fairly investigated in the literature under the relaying strategies of amplify-andforward (AF) [6], [7] and decode-and-forward (DF) [8], [9].

The aforementioned efforts considered conventional halfduplex relaying (HDR). However, HDR suffers from a spectral efficiency loss when compared to direct transmission (DT) due to its time-orthogonal relay listening/forwarding. More recently, full-duplex communications experienced serious developments that brought full-duplex node prototypes into reality [10], [11]. These developments motivated further research in cooperative contexts where simultaneous listening/forwarding is allowed by full-duplex relaying $(F D R)$, thereby eliminating the HDR rate loss. Recent efforts were directed to study FDR in several directions, for instance, in CRNs [12], [13], and with relay selection [14]-[24]. These research endeavors and practical potentials motivated the International Telecommunication Union (ITU) to recommend FDR for employment in fifthgeneration (5G) cellular networks [25], [26]. When underlay settings are particularly addressed, FDR offers a real potential to boost the spectral efficiency of the secondary system as compared to HDR. Meanwhile, another challenge exists, where the allowable interference threshold on the primary receiver is now imposed on the superposition of the simultaneous transmissions from the source and the full-duplex relay.

\section{A. Related Work}

In [13], an underlay setting is studied where a secondary network coexists with a primary network under an interference constraint. The secondary system comprises a source, a destination and a full-duplex DF relay. Under the aforementioned interference constraint, optimal source/relay power allocation is investigated to minimize the end-to-end outage probability. In [14], Krikidis et al. studied the outage performance of AF full-duplex relay selection (FDRS) with residual selfinterference (RSI) for coverage extension, where no direct link exists between the source and the destination. In this work, we focus our attention on DF relaying, also for more general settings when a direct link may exist, covering both coverage extensions and throughput enhancement purposes. 
For DF networks, FDRS is briefly discussed in [16], however only the simple case of non-fading RSI link is considered, and the adverse effect of the interfering direct link in FDR was not accounted for. In [17], DF FDRS is studied under Rayleigh fading while accounting for the effect of a fading RSI link, yet the direct link was assumed unavailable. In [18], the authors have studied DF FDRS under Rayleigh fading while jointly taking into account the effects of fading RSI and direct links, in an underlay setting where a primary receiver imposes an interference constraint. The authors also analyzed the performance of DF FDRS in [19] under Nakagami- $m$ fading in the absence of spectrum sharing. A similar setting was also briefly discussed in [20] for Nakagami- $m$ fading, where the relay selection is performed based only on the strength of the self-interference channel. However, the direct sourcedestination link was assumed unavailable in [20], and also no analysis was provided. The results in [17] have been recently extended in [21] to Nakagami- $m$ fading scenarios when no direct link exists in the presence of a primary receiver. Also recently, [22] has studied the extension of the selective DF (SDF) FDR protocol proposed by the authors in [27], [28] to the FDRS setting under Nakagami- $m$ fading without spectrum sharing constraints. The work in [23] considered partial relay selection, based on the link strengths of only the first hop, under Rayleigh fading and without a direct source-destination link when multiple primary users exist. The interested reader is also referred to [29] for a recent comprehensive survey, listing the latest research endeavors on the topic.

Despite its anticipated performance merits, the incorporation of relay selection techniques into cognitive FDR networks remained untackled to the best of the authors' knowledge till the work by the authors in [18]. In this work, which subsumes the results reported in [18] as a special case, we aim at bridging this gap by introducing relay selection to cognitive FDR networks and analyzing its offered outage probability and throughput performance, also in the presence of a direct source-destination link. In underlay settings with FDR, the secondary system's performance can be seriously limited since the superimposed source and relay interference components should not violate the existing interference threshold. This comes in contrast to HDR where the threshold is imposed on each transmit node at a time. In this regard, relay selection techniques can offer an adequate solution to boost the secondary network throughput. Comparing FDRS to classical half-duplex relay selection (HDRS), now from an interference perspective, such a source/relay interference superposition is expected to yield a narrower feasible set of full-duplex relays. That being said, a tradeoff exists between the gained spectral efficiency and the shrinked feasible relay set size when migrating to FDRS in underlay settings. It is then interesting to numerically compare the overall end-to-end throughput of the two duplexing approaches. Moreover, in fullduplex systems, the analysis of the end-to-end diversity gain highly depends on the proper modeling of the RSI link. Earlier cancellation techniques suggested an RSI link gain model that scales linearly with the transmit power of the full-duplex node [30]. More recent passive and active cancellation techniques were analyzed in [31]-[33]. These techniques propose a dif- ferent scaling trend, where the adaptive cancellation methods may actually succeed to yield a nearly constant average RSI link gain while varying the relay power. Considering a model that spans the whole range of linear, sublinear and constant power scaling is hence crucial for FDR evaluation. This general model has been adopted in the recent literature [28], [34].

\section{B. Contribution}

Our contribution in this paper is summarized as follows: ${ }^{1}$

- We derive the exact cumulative distribution function (CDF) for the end-to-end signal-to-interference-plusnoise ratio (SINR) of opportunistic DF FDRS in the presence/absence of a direct source-destination link in addition to a spectrum-sharing constraint, where all links experience Nakagami- $m$ fading. The end-to-end SINR $\mathrm{CDF}$ is obtained and compared for two cooperation classes, namely, 1) multihop cooperation [35], where information is distilled only from the relayed path, and 2) selective block cooperation [22], [27], [28], where the direct information from the source is additionally exploited at the destination. When a direct sourcedestination link exists, the SINR values of the different relaying paths become mutually dependent, which we account for in the analysis. Also, DT can take place if the direct link supports higher instantaneous rate than those offered through relaying.

- From the derived CDF expressions, we analyze the diversity gains offered by FDRS in the presence/absence of direct source-destination link. When the adopted RSI link gain model is sublinear in the relay power, which agrees with recent research findings [31]-[33], we show that a remarkable diversity can be achieved even for the simpler multihop cooperation protocols which treat the direct link as interference to the relayed paths. This result gets around a known drawback in FDR in the literature where a zero diversity order, i.e., an error floor, is encountered even in the absence of RSI due to the interfering direct link effect. We also show that hybrid multihop-based FDRS/DT achieves the same diversity order of the SDF-FDRS. Since multihop cooperation adopts a simpler transmission/decoding approach, it may be favored over the selective cooperation class when decoding complexity represents a major concern.

- We compare the performance of the considered fullduplex cooperative schemes to that of their half-duplex counterparts for different scaling trends of the RSI, also in the presence of a primary receiver.

\footnotetext{
${ }^{1}$ While this work was in progress, preliminary results were accepted for presentation in IEEE ICC Wkshps'15 [18] for the spectrum-sharing setting under Rayleigh fading, and in IEEE CAMAD'15 [19] for the non-spectrumsharing setting under Nakagami- $m$ fading. The currently presented work reports the full results for the spectrum-sharing setting under Nakagami- $m$ fading which subsumes the results in [18] and [19] as special cases. Also presented is the diversity analysis of the FDRS protocols, as well as the relative performance to the HDRS protocols.
} 


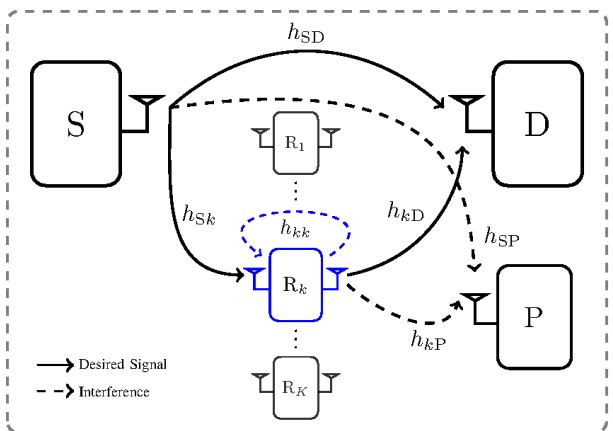

Fig. 1: A spectrum-sharing network with full-duplex relaying.

\section{Paper Outline}

The rest of the paper is organized as follows. In section II, we explain the adopted system model and its main assumptions. The end-to-end SINR CDF is derived in section III, which readily yields the outage probability. Section IV presents the derived SINR CDF/outage probability expressions when a primary user exists. The diversity order is derived in section $\mathrm{V}$ under Rayleigh fading. Numerical results are presented in section VI, while conclusions are drawn in section VII.

\section{SYSTEM MODEL}

We consider the underlay setting depicted in Fig. 1, where a secondary source (S) communicates with a secondary destination (D) in the presence of a primary receiver (P). In agreement with similar studies of underlay settings, for instance in [8], [21], [24] and the references therein, the primary source is assumed to be far enough that its interference on the secondary system can be reasonably ignored. The direct secondary $\mathrm{S}-\mathrm{D}$ link is assumed of a relatively weak gain due to distance and shadowing effects. Hence, a full-duplex DF relay is utilized to assist the end-to-end secondary communication, taking the interference constraint on the primary receiver into account. Although FDR can offer higher spectral efficiency when compared to HDR, an additional challenge is posed in cognitive settings where higher interference levels may be experienced by the primary user due to simultaneous source/relay transmissions. Also, FDR suffers from an RSI level which imposes another communication challenge.

In this regard, relay selection offers an adequate mean to alleviate the aforementioned interference problems. Specifically, the secondary system has the additional freedom to select one out of $K$ full-duplex relays, $\mathcal{R}=\left\{\mathrm{R}_{1}, \mathrm{R}_{2}, \cdots, \mathrm{R}_{K}\right\}$. In underlay scenarios, selection remains of a particular payoff as it allows picking the best relay while abiding by the primaryimposed interference limit.

The relay selection process can be governed either via a centralized [5] or a distributed fashion [4], with the latter being more challenging in general. A distributed scheme can be adopted here, where each relay estimates the receive channel state information (CSI) of its own incoming link from the source. Also, it estimates the CSI of its outgoing link from the previously received acknowledgement (ACK) packet from the destination while leveraging channel reciprocity. In addition, each relay locally estimates its own instantaneous RSI link power gain, $g_{k k}=\left|h_{k k}\right|^{2}$, using typical signal integrators found in energy detectors with known pilot signals at the beginning of each channel block. Accordingly, a local reverse timer is triggered at each relay based on its end-to-end channel quality, where the better the channel condition is, the faster the timer expires. All relays operate in a listening mode within this phase, allowing the relay with the first expired timer to transmit, while all other relays accordingly backoff. Such a reverse timer/backoff method is widely known for distributed wireless multiple access purposes [4]. When a direct $\mathrm{S}-\mathrm{D}$ link exists, the destination is assumed to additionally feedback its CSI within the ACK packet of the previous transmission. Since an underlay setting is considered, no cooperation is assumed between the primary and secondary networks. The relays are assumed to overhear the ACK packets sent from the primary receiver to its own source, and hence, locally estimate its channel, also utilizing channel reciprocity. It can be noticed that only local link strengths are required here, aided by simple feedback from the destination which does not scale with the number of relays. It should be also noted here that this knowledge is only required for the relay selection at the upper layers of the communication protocol stack. Such a knowledge is not required for the communication process itself over the physical layer. Hence, further enhancements to the relay selection process at the upper layers remain possible, however, this lies beyond the scope of the current paper.

\section{A. Channel Model}

The fading coefficient of the $i-j$ link is denoted by $h_{i j}$, for $i \in\{\mathrm{S}, \mathcal{K}\}$ and $j \in\{\mathcal{K}, \mathrm{D}, \mathrm{P}\}$, where $\mathcal{K}=\{1,2, \cdots, K\}$ denotes the set of relay indices. The $i-j$ link gain is denoted by $g_{i j}=\left|h_{i j}\right|^{2}$. The relays operate in full-duplex mode where simultaneous listening/forwarding is allowed with an introduced level of loopback interference. The link gain $h_{k k}, \forall k \in \mathcal{K}$, represents the RSI after undergoing all possible isolation and cancellation techniques, as for instance in [36]. All channels are assumed to follow a block fading model, where $h_{i j}$ remains constant over one block, and varies independently from a block to another according to a Nakagami$m$ fading model with shape parameter $m_{i j}$ and average power $\mathbb{E}\left\{g_{i j}\right\}=\pi_{i j}$ [28]. Accordingly, $g_{i j}$ is a Gamma-distributed random variable (RV) with shape parameter $m_{i j}$ and scale parameter $\theta_{i j}=\frac{\pi_{i j}}{m_{i j}}$, for which we use the shorthand notation $g_{i j} \sim \mathcal{G}\left(m_{i j}, \theta_{i j}\right)$. For a Gamma $\operatorname{RV} X \sim \mathcal{G}(m, \theta)$, the probability density function (PDF) and the CDF are given by

$$
f_{X}(x ; m, \theta)=\frac{x^{m-1} e^{-\frac{x}{\theta}}}{\boldsymbol{\Gamma}(m) \theta^{m}} \text { and } F_{X}(x ; m, \theta)=\frac{\gamma\left(m, \frac{x}{\theta}\right)}{\boldsymbol{\Gamma}(m)},
$$

respectively, where $\gamma(a, b)=\int_{0}^{b} t^{a-1} e^{-t} d t$ denotes the lower incomplete Gamma function, and $\boldsymbol{\Gamma}(a)$ denotes the Gamma function [37]. All channel fading gains are assumed to be mutually independent. The source and the $k^{\text {th }}$ relay powers are denoted by $P_{\mathrm{S}}$ and $P_{k}$, respectively. Also, $n_{k}$ and $n_{\mathrm{D}}$ denote the complex additive white Gaussian noise (AWGN) components at the $k^{\text {th }}$ relay and the destination, with variance $\sigma_{k}^{2}$ and $\sigma_{\mathrm{D}}^{2}$, respectively. 
As commonly assumed in the literature, for instance in [8], [20]-[23], we consider the relays to be clustered somewhere between $\mathrm{S}$ and $\mathrm{D}$. Hence, the distances among the relays are much shorter than those between the relays and the source/destination. Thus, it is reasonable to assume the symmetric scenario where all source-relay links have an average gain of $\mathbb{E}\left\{g_{\mathrm{S} k}\right\}=\pi_{\mathrm{SR}}$, while all relay-destination links have an average gain of $\mathbb{E}\left\{g_{k \mathrm{D}}\right\}=\pi_{\mathrm{RD}}, \forall k \in \mathcal{K}$. All relays are assumed to have RSI links and those interfering on $\mathrm{P}$ with the same average gains, $\mathbb{E}\left\{g_{k k}\right\}=\pi_{\mathrm{RR}}$ and $\mathbb{E}\left\{g_{k \mathrm{P}}\right\}=\pi_{\mathrm{RP}}$, respectively. Further, it is assumed that $m_{\mathrm{S} k}=m_{\mathrm{SR}}, m_{k \mathrm{D}}=$ $m_{\mathrm{RD}}, m_{k k}=m_{\mathrm{RR}}$ and $m_{k \mathrm{P}}=m_{\mathrm{RP}}$, for all $k \in \mathcal{K}$. It follows that $\theta_{\mathrm{S} k}=\theta_{\mathrm{SR}}, \theta_{k \mathrm{D}}=\theta_{\mathrm{RD}}, \theta_{k k}=\theta_{\mathrm{RR}}$ and $\theta_{k \mathrm{P}}=\theta_{\mathrm{RP}}$ for all $k \in \mathcal{K}$. Although the analysis of asymmetric scenarios remains possible, such assumptions allow for simpler final expressions, yet maintain the same diversity order of the system. Finally, we assume that $\sigma_{k}^{2}=\sigma_{\mathrm{D}}^{2}=1$, while all relays have the same transmit power $P_{k}=P_{\mathrm{R}}$.

\section{B. Signal Model}

When the $k^{\text {th }}$ relay is selected, the received signals at the $k^{\text {th }}$ relay and destination at time $t$ are given, respectively, by

$$
\begin{aligned}
y_{k}[t] & =\sqrt{P_{\mathrm{S}}} h_{\mathrm{S} k} x_{\mathrm{S}}[t]+\sqrt{P_{\mathrm{R}}^{\delta}} h_{k k} x_{k}[t]+n_{k}[t], \\
y_{\mathrm{D}}[t] & =\sqrt{P_{\mathrm{R}}} h_{k \mathrm{D}} x_{k}[t]+\sqrt{P_{\mathrm{S}}} h_{\mathrm{SD}} x_{\mathrm{S}}[t]+n_{\mathrm{D}}[t],
\end{aligned}
$$

where $P_{i}$ and $x_{i}[t]$ denote the transmit power and the transmit symbol at time $t$ at node $i \in\{\mathrm{S}, \mathrm{R}\}$, respectively, while $n_{i}[t]$ represents the AWGN component at node $i \in\{\mathrm{R}, \mathrm{D}\}$ at time $t$. It is assumed that the RSI term proportionally scales with $P_{\mathrm{R}}^{\delta}$, where the exponent $0 \leq \delta \leq 1$ depends on the employed cancellation techniques [30]-[33], covering the range from constant to linear scaling with the relay power [34].

Due to the source/relay asynchronous transmissions, the signal transmitted by the relay (source) is considered in this class as an additional noise term at the relay (destination) as commonly treated in the related literature [36]. Accordingly, the received SINRs at the $k^{\text {th }}$ DF relay and at the destination are given respectively by

$$
\gamma_{\mathrm{S} k}=\frac{P_{\mathrm{S}} g_{\mathrm{S} k}}{P_{\mathrm{R}}^{\delta} g_{k k}+1} \text { and } \gamma_{k \mathrm{D}}=\frac{P_{\mathrm{R}} g_{k \mathrm{D}}}{P_{\mathrm{S}} g_{\mathrm{SD}}+1} .
$$

Since only the multi-hop path information is utilized, we refer to this protocol, as previously used in the literature [28], [38], as the multi-hop decode-and-forward (MHDF) protocol.

Underlay Cognitive Setting: In an underlay setting, the introduced interference level on $\mathrm{P}$ is constrained not to exceed a certain threshold, $I_{\mathrm{th}}$. Thus, when no relays are selected while a direct $\mathrm{S}-\mathrm{D}$ link exists, the interference constraint is simply given by

$$
I_{\mathrm{SP}} \leq I_{\mathrm{th}}
$$

When the $k^{\text {th }}$ DF relay is selected, the constraint is given by

$$
I_{k}=I_{\mathrm{SP}}+I_{k \mathrm{P}} \leq I_{\mathrm{th}},
$$

where $I_{\mathrm{SP}}=P_{\mathrm{S}} g_{\mathrm{SP}}$ and $I_{k \mathrm{P}}=P_{\mathrm{R}} g_{k \mathrm{P}}$ are the interference components imposed on the primary receiver due to the source and the $k^{\text {th }}$ relay, respectively, while $I_{k}$ denotes their sum. We assume that the secondary source additionally announces the value of $I_{\mathrm{th}}-I_{\mathrm{SP}}$ within its frame structure to all the relays. We note here that $P_{\mathrm{S}}$ and $P_{\mathrm{R}}$ are fixed values. It hence suffices for each relay to know $P_{\mathrm{S}}$ and the dictated $I_{\mathrm{th}}$ and $I_{\mathrm{th}}-I_{\mathrm{SP}}$, in addition to the channel gains as discussed earlier, to locally decide its own feasibility. The same applies to the source by itself, since no source/relay power-level coordination is assumed.

\section{Full-Duplex Relay Selection}

We first derive the exact end-to-end SINR CDF for three MHDF scenarios; 1) no direct link (NDL), 2) interfering direct link (IDL) where a direct link exists, yet it is treated as mere interference, and 3) hybrid IDL/DT. By selecting the relay offering the highest SINR among $K$ DF relays, the resulting end-to-end SINR is given by

$$
\gamma_{\mathrm{e} 2 \mathrm{e}}=\max _{k \in \mathcal{K}}\left\{\gamma_{k}\right\}
$$

where

$$
\gamma_{k}=\min \left\{\gamma_{\mathrm{S} k}, \gamma_{k \mathrm{D}}\right\}
$$

represents the SINR over the path passing via the $k^{\text {th }}$ DF relay. For the MHDF-NDL scenario, $\gamma_{k \mathrm{D}}$ is calculated for $\gamma_{\mathrm{SD}}=$ $P_{\mathrm{S}} g_{\mathrm{SD}}=0$. The expression in (7) also applies to the MHDFIDL scenario. Alternatively, if DT is taken into account as a possible diversity branch, the end-to-end SINR of the MHDFIDL/DT protocol is given by

$$
\gamma_{\mathrm{e} 2 \mathrm{e}}=\max \left\{\max _{k \in \mathcal{K}}\left\{\gamma_{k}\right\}, \gamma_{\mathrm{SD}}\right\} .
$$

\section{A. CDF of Link SINRs}

1) First Hop: According to the channel model explained in Section II-A, the CDF of the SINR pertaining to the first hop of the $k^{t h}$ path is given as

$$
F_{\gamma_{\mathrm{S} k}}(x)=F_{Z}\left(x ; \boldsymbol{p}_{1}\right),
$$

where $\boldsymbol{p}_{1}=\left(m_{\mathrm{SR}}, P_{\mathrm{S}} \theta_{\mathrm{SR}}, m_{\mathrm{RR}}, P_{\mathrm{R}}^{\delta} \theta_{\mathrm{RR}}\right)$, while $F_{Z}(x ; \boldsymbol{p})$ is given in the following remark [39], [40].

Remark 1 (CDF of $\left.Z=\frac{X_{1}}{X_{2}+1}\right)$ : The CDF of $Z=\frac{X_{1}}{X_{2}+1}$, where $X_{i} \sim \mathcal{G}\left(m_{i}, \theta_{i}\right)$, for $i \in\{1,2\}$, are independent but not identically distributed (i.n.i.d.) RVs, for general real-valued $m_{1} \geq \frac{1}{2}$ and integer-valued $m_{2} \geq 1$, is given by [39], [40]:

$$
F_{Z}(z ; \boldsymbol{p})=\frac{\boldsymbol{\gamma}\left(m_{1}, \frac{z}{\theta_{1}}\right)}{\boldsymbol{\Gamma}\left(m_{1}\right)}+B \sum_{k=0}^{m_{2}-1} \frac{c^{-d}}{\theta_{2}{ }^{k}} \boldsymbol{W}_{a, b}(c),
$$

where $\boldsymbol{p}=\left(m_{1}, \theta_{1}, m_{2}, \theta_{2}\right)$ is a vector of distribution parameters, $\gamma(\alpha, \beta)=\int_{0}^{\beta} t^{\alpha-1} e^{-t} d t$ is the lower incomplete Gamma function, $\boldsymbol{W}_{a, b}(c)$ is the Whittaker function [37, Eq. 13.1.33], $a=\frac{m_{1}-k-1}{2}, b=\frac{-m_{1}-k}{2}, c=\frac{z}{\theta_{1}}+\frac{1}{\theta_{2}}, d=\frac{m_{1}+k+1}{2}$ and

$$
B=\frac{\exp \left(-\frac{1}{2}\left(\frac{z}{\theta_{1}}-\frac{1}{\theta_{2}}\right)\right)}{\boldsymbol{\Gamma}\left(m_{1}\right)}\left(\frac{z}{\theta_{1}}\right)^{m_{1}} .
$$


2) Second Hop: Since the direct link signal-to-noise ratio (SNR), $\gamma_{\mathrm{SD}}$, is a common RV among all the multi-hop paths, all the second-hop gains are clearly correlated. However, they are conditionally independent given $\gamma_{\mathrm{SD}}=\beta$. Thus, in the presence of a direct link, we are only interested in the conditional distributions of the second-hop gains given $\gamma_{\mathrm{SD}}=\beta$, which follow $\gamma_{k \mathrm{D}} \mid \gamma_{\mathrm{SD}} \sim \mathcal{G}\left(m_{\mathrm{RD}}, \frac{P_{\mathrm{R}} \theta_{\mathrm{RD}}}{\beta+1}\right), \forall k \in \mathcal{K}$. On the other hand, when no direct link exists, it is clear that $\gamma_{k \mathrm{D}} \mid \gamma_{\mathrm{SD}} \sim \mathcal{G}\left(m_{\mathrm{RD}}, P_{\mathrm{R}} \theta_{\mathrm{RD}}\right)$.

3) The $k^{\text {th }}$ Multi-Hop Path: According to (8) and with the above-mentioned distributions of the hop SINRs, the conditional CDF of the SINR over the $k^{t h}$ path given the direct link SNR $\gamma_{\mathrm{SD}}=\beta$ is given by

$$
F_{\gamma_{k} \mid \gamma_{\mathrm{SD}}}(x \mid \beta)=1-\overline{F_{Z}}\left(x ; \boldsymbol{p}_{1}\right) \overline{F_{X}}\left(x ; m_{\mathrm{RD}}, \frac{P_{\mathrm{R}} \theta_{\mathrm{RD}}}{\beta+1}\right) \text {, }
$$

where $\bar{F}(\cdot)=1-F(\cdot)$ denotes the complementary CDF. It is clear that in the absence of a direct link, the CDF of the $k^{t h}$ path SINR is given by

$$
F_{\gamma_{k}}(x)=1-\overline{F_{Z}}\left(x ; \boldsymbol{p}_{1}\right) \overline{F_{X}}\left(x ; m_{\mathrm{RD}}, P_{\mathrm{R}} \theta_{\mathrm{RD}}\right) .
$$

\section{B. CDF of End-to-End SINR}

1) NDL: In the absence of a direct link, the end-to-end SINR $\mathrm{CDF}$ has the following simple form [21]:

$$
F_{\gamma_{\mathrm{e} 2 \mathrm{e}}}^{\mathrm{NDL}}(x)=\left(1-\overline{F_{Z}}\left(x ; \boldsymbol{p}_{1}\right) \overline{F_{X}}\left(x ; m_{\mathrm{RD}}, P_{\mathrm{R}} \theta_{\mathrm{RD}}\right)\right)^{K},
$$

which is explicitly given in (16) for real-valued $m_{\mathrm{SR}}$ and $m_{\mathrm{RD}}$, and integer-valued $m_{\mathrm{RR}}$.

2) IDL: When a direct link exists, yet treated as interference to the multi-hop paths, the end-to-end SINR CDF is given by

$$
\begin{aligned}
F_{\gamma_{\mathrm{e} 2 \mathrm{e}}}^{\mathrm{IDL}}(x) & =\int_{0}^{\infty} F_{\gamma_{k} \mid \gamma_{\mathrm{SD}}}(x \mid \beta)^{K} f_{x}\left(\beta ; m_{\mathrm{SD}}, P_{\mathrm{S}} \theta_{\mathrm{SD}}\right) \mathrm{d} \beta \\
& =\sum_{k=0}^{K} C(x, k) \mathcal{I}_{1}
\end{aligned}
$$

$$
\begin{aligned}
& \mathcal{I}_{1}=\int_{0}^{\infty} \frac{\boldsymbol{\Gamma}\left(m_{\mathrm{RD}}, \frac{x(\beta+1)}{P_{\mathrm{R}} \theta_{\mathrm{RD}}}\right)^{k}}{\boldsymbol{\Gamma}\left(m_{\mathrm{RD}}\right)^{k}} \beta^{m_{\mathrm{SD}}-1} e^{-\frac{\beta}{P_{\mathrm{S}} \theta_{\mathrm{SD}}}} \mathrm{d} \beta, \\
& C(x, k)=\left(\begin{array}{c}
K \\
k
\end{array}\right) \frac{\left(-\overline{F_{Z}}\left(x ; \boldsymbol{p}_{1}\right)\right)^{k}}{\boldsymbol{\Gamma}\left(m_{\mathrm{SD}}\right)\left(P_{\mathrm{S}} \theta_{\mathrm{SD}}\right)^{m_{\mathrm{SD}}}},
\end{aligned}
$$

where the binomial expansion is utilized in the last step. We are now interested in solving the integral $\mathcal{I}_{1}$. Using the finite series expansion of the upper incomplete Gamma function in [41, Eq. 8.352-2], for integer values of $m_{\mathrm{RD}}$, we get

$$
\begin{aligned}
\mathcal{I}_{1} & =\int_{0}^{\infty}\left(e^{-\frac{x(\beta+1)}{P_{\mathrm{R}} \theta_{\mathrm{RD}}}} \sum_{n=1}^{m_{\mathrm{RD}}} \frac{\left(\frac{x(\beta+1)}{P_{\mathrm{R}} \theta_{\mathrm{RD}}}\right)^{n-1}}{\Gamma(n)}\right)^{k} \beta^{m_{\mathrm{SD}}-1} e^{-\frac{\beta}{P_{\mathrm{S}} \theta_{\mathrm{SD}}}} \mathrm{d} \beta \\
= & e^{-\frac{x k}{P_{\mathrm{R}} \theta_{\mathrm{RD}}}} \sum_{\sum_{n=1}^{m} k_{\mathrm{RD}}=k} C_{\left\{k_{n}\right\}} \mathcal{I}_{2},
\end{aligned}
$$

where

$$
\begin{aligned}
\mathcal{I}_{2} & =\int_{0}^{\infty}(\beta+1)^{D_{\left\{k_{n}\right\}} \beta^{m_{\mathrm{SD}}-1} e^{-\beta \eta_{k}} \mathrm{~d} \beta,} \\
\eta_{k} & =\left(\frac{1}{P_{\mathrm{S}} \theta_{\mathrm{SD}}}+\frac{x k}{P_{\mathrm{R}} \theta_{\mathrm{RD}}}\right), \\
C_{\left\{k_{n}\right\}} & =\frac{\boldsymbol{\Gamma}(k+1)\left(\frac{x}{P_{\mathrm{R}} \theta_{\mathrm{RD}}}\right)^{\sum_{n=1}^{m_{\mathrm{RD}}} k_{n}(n-1)}}{\prod_{n=1}^{m_{\mathrm{RD}}}\left(\boldsymbol{\Gamma}\left(k_{n}+1\right) \Gamma(n)^{k_{n}}\right)}, \\
D_{\left\{k_{n}\right\}} & =\sum_{n=1}^{m_{\mathrm{RD}}} k_{n}(n-1),
\end{aligned}
$$

with (18) obtained via the multinomial theorem [37, Section 24.1.2]. By substitution of variables, $y=\beta+1, \mathcal{I}_{2}$ in (19) is the Riemann-Liouville integral in [41, Eq. 3.383-4]. Hence,

$$
\begin{aligned}
\mathcal{I}_{2}= & e^{\eta_{k}} \int_{1}^{\infty} y^{D_{\left\{k_{n}\right\}}}(y-1)^{m_{\mathrm{SD}}-1} e^{-y \eta_{k}} \mathrm{~d} y \\
= & e^{\frac{\eta_{k}}{2}} \eta_{k}^{-\frac{m_{\mathrm{SD}}+D_{\left\{k_{n}\right\}}+1}{2}} \boldsymbol{\Gamma}\left(m_{\mathrm{SD}}\right) \\
& \times \boldsymbol{W}_{\frac{D_{\left\{k_{n}\right\}}-m_{\mathrm{SD}}+1}{2}},-\frac{m_{\mathrm{SD}}+D_{\left\{k_{n}\right\}}}{2}\left(\eta_{k}\right) .
\end{aligned}
$$

Accordingly, $F_{\gamma_{\mathrm{e} 2 \mathrm{e}}}^{\mathrm{IDL}}(x)$ is given by (24) for integer-valued $m_{\mathrm{RR}}$ and $m_{\mathrm{RD}}$, and real-valued $m_{\mathrm{SR}}$ and $m_{\mathrm{SD}}$.

3) Hybrid IDL/DT: When the $\mathrm{S}-\mathrm{D}$ link is used as an additional diversity path, $\gamma_{\mathrm{SD}}=\beta$ is bounded above by $x$. Hence, the CDF expression is similar to that of the IDL case, however with the upper integration limit changed to $x$ to be

$$
F_{\gamma_{\mathrm{e} 2 \mathrm{e}}}^{\mathrm{IDL} / \mathrm{DT}}(x)=\int_{0}^{x} F_{\gamma_{k} \mid \gamma_{\mathrm{SD}}}(x \mid \beta)^{K} f_{x}\left(\beta ; m_{\mathrm{SD}}, P_{\mathrm{S}} \theta_{\mathrm{SD}}\right) \mathrm{d} \beta .
$$

The evaluation of such an integral follows the same steps, except that the integral $\mathcal{I}_{2}$ is changed to

$$
\begin{aligned}
\tilde{\mathcal{I}}_{2} & =\int_{0}^{x}(\beta+1)^{D_{\left\{k_{n}\right\}} \beta^{m_{\mathrm{SD}}-1} e^{-\beta \eta_{k}} \mathrm{~d} \beta} \\
& =\sum_{r=0}^{D_{\left\{k_{n}\right\}}}\left(\begin{array}{c}
D_{\left\{k_{n}\right\}} \\
r
\end{array}\right) \int_{0}^{x} \beta^{r+m_{\mathrm{SD}}-1} e^{-\beta \eta_{k}} \mathrm{~d} \beta \\
& =\sum_{r=0}^{D_{\left\{k_{n}\right\}}}\left(\begin{array}{c}
D_{\left\{k_{n}\right\}} \\
r
\end{array}\right) \eta_{k}^{-\left(r+m_{\mathrm{SD}}\right)} \gamma\left(r+m_{\mathrm{SD}}, x \eta_{k}\right),
\end{aligned}
$$

for integer-valued $m_{\mathrm{SD}}$ due to the use of the binomial theorem, with the last integral evaluated using [41, Eq. 3.381-1]. Hence, $F_{\gamma_{\mathrm{e} 2 \mathrm{e}}}^{\mathrm{IDL}}(x)$ is finally given by (27) for real-valued $m_{\mathrm{SR}}$ and integer-valued $m_{\mathrm{RR}}, m_{\mathrm{RD}}$ and $m_{\mathrm{SD}}$.

4) Selective decode-and-forward: Unlike the previous nonselective multi-hop protocols, selective block full-duplex cooperation is recently studied in [27], [28]. In this class, both the multi-hop path and the direct path are simultaneously exploited as two information-bearing paths. In order to leverage the information in the two paths in the presence of their relative delay, a block transmission scheme is adopted [27]. When SDF is considered, the instantaneous SINR in the first hop via the $k^{\text {th }}$ relay, its virtual multiple-input single-output (MISO) channel with the source and the $k^{t h}$ relay as the transmitter 


$$
\begin{aligned}
& F_{\gamma_{\mathrm{e} 2 \mathrm{e}}}^{\mathrm{IDL}}(x)=\sum_{k=0}^{K}\left(\begin{array}{c}
K \\
k
\end{array}\right) \frac{e^{\left(\frac{1}{2 P_{\mathrm{S}}{ }_{\mathrm{SD}}}-\frac{x k}{2 P_{\mathrm{R}} \mathrm{RD}}\right)}}{\left(P_{\mathrm{S}} \theta_{\mathrm{SD}}\right)^{m_{\mathrm{SD}}} \boldsymbol{\Gamma}\left(m_{\mathrm{SR}}\right)^{k}}\left(e^{-\frac{1}{2}\left(\frac{x}{P_{\mathrm{S}} \theta_{\mathrm{SR}}}-\frac{1}{P_{\mathrm{R}}^{\delta} \theta_{\mathrm{RR}}}\right.}\right)\left(\frac{x}{P_{\mathrm{S}} \theta_{\mathrm{SR}}}\right)^{m_{\mathrm{SR}}} \\
& \left.\times \sum_{l=0}^{m_{\mathrm{RR}}-1} \frac{\left(\frac{x}{P_{\mathrm{S}} \theta_{\mathrm{SR}}}+\frac{1}{P_{\mathrm{R}}^{\delta} \theta_{\mathrm{RR}}}\right)^{-\frac{m_{\mathrm{SR}}+l+1}{2}}}{\left(P_{\mathrm{R}}^{\delta} \theta_{\mathrm{RR}}\right)^{l}} \boldsymbol{W}_{\frac{m_{\mathrm{SR}}-l-1}{2}, \frac{-m_{\mathrm{SR}}-l}{2}}\left(\frac{x}{P_{\mathrm{S}} \theta_{\mathrm{SR}}}+\frac{1}{P_{\mathrm{R}}^{\delta} \theta_{\mathrm{RR}}}\right)-\boldsymbol{\Gamma}\left(m_{\mathrm{SR}}, \frac{x}{P_{\mathrm{S}} \theta_{\mathrm{SR}}}\right)\right)^{k} \\
& \times \sum_{\sum_{n=1}^{m_{\mathrm{RD}}} k_{n}=k} \frac{\boldsymbol{\Gamma}(k+1)\left(\frac{x}{P_{\mathrm{R}} \theta_{\mathrm{RD}}}\right)^{\sum_{n=1}^{m_{\mathrm{RD}}} k_{n}(n-1)}}{\prod_{n=1}^{m_{\mathrm{RD}}}\left(\boldsymbol{\Gamma}\left(k_{n}+1\right) \boldsymbol{\Gamma}(n)^{k_{n}}\right)} \eta_{k}^{-\frac{m_{\mathrm{SD}}+\sum_{n=1}^{m_{\mathrm{RD}} k_{n}(n-1)+1}}{2}} \boldsymbol{W}_{\frac{\sum_{n=1}^{m_{\mathrm{RD}} k_{n}(n-1)-m_{\mathrm{SD}}+1}}{2},-\frac{m_{\mathrm{SD}}+\sum_{n=1}^{m_{\mathrm{RD}}} k_{n}(n-1)}{2}}\left(\eta_{k}\right) .
\end{aligned}
$$

$$
\begin{aligned}
& F_{\gamma_{\mathrm{e} 2 \mathrm{e}}}^{\mathrm{IDL} / \mathrm{DT}}(x)=\sum_{k=0}^{K}\left(\begin{array}{c}
K \\
k
\end{array}\right) \frac{e^{-\frac{x k}{P_{\mathrm{R}} \theta_{\mathrm{RD}}}}}{\boldsymbol{\Gamma}\left(m_{\mathrm{SD}}\right)\left(P_{\mathrm{S}} \theta_{\mathrm{SD}}\right)^{m_{\mathrm{SD}}} \boldsymbol{\Gamma}\left(m_{\mathrm{SR}}\right)^{k}}\left(e^{-\frac{1}{2}\left(\frac{x}{P_{\mathrm{S}} \theta_{\mathrm{SR}}}-\frac{1}{P_{\mathrm{R}}^{\delta} \theta_{\mathrm{RR}}}\right.}\right)\left(\frac{x}{P_{\mathrm{S}} \theta_{\mathrm{SR}}}\right)^{m_{\mathrm{SR}}} \\
& \left.\times \sum_{l=0}^{m_{\mathrm{RR}}-1} \frac{\left(\frac{x}{P_{\mathrm{S}} \theta_{\mathrm{SR}}}+\frac{1}{P_{\mathrm{R}}^{\delta} \theta_{\mathrm{RR}}}\right)^{-\frac{m_{\mathrm{SR}}+l+1}{2}}}{\left(P_{\mathrm{R}}^{\delta} \theta_{\mathrm{RR}}\right)^{l}} \boldsymbol{W}_{\frac{m_{\mathrm{SR}}-l-1}{2}, \frac{-m_{\mathrm{SR}}-l}{2}}\left(\frac{x}{P_{\mathrm{S}} \theta_{\mathrm{SR}}}+\frac{1}{P_{\mathrm{R}}^{\delta} \theta_{\mathrm{RR}}}\right)-\boldsymbol{\Gamma}\left(m_{\mathrm{SR}}, \frac{x}{P_{\mathrm{S}} \theta_{\mathrm{SR}}}\right)\right)^{k} \\
& \times \sum_{\sum_{n=1}^{m_{\mathrm{RD}}} k_{n}=k} \frac{\boldsymbol{\Gamma}(k+1)\left(\frac{x}{P_{\mathrm{R}} \theta_{\mathrm{RD}}}\right)^{\sum_{n=1}^{m_{\mathrm{RD}}} k_{n}(n-1)}}{\prod_{n=1}^{m_{\mathrm{RD}}}\left(\boldsymbol{\Gamma}\left(k_{n}+1\right) \boldsymbol{\Gamma}(n)^{k_{n}}\right)} \sum_{r=0}^{\sum_{n=1}^{m_{\mathrm{RD}}} \sum_{n}(n-1)}\left(\begin{array}{c}
\sum_{n=1}^{m_{\mathrm{RD}}} k_{n}(n-1) \\
r
\end{array}\right) \eta_{k}^{-\left(r+m_{\mathrm{SD}}\right)} \gamma\left(r+m_{\mathrm{SD}}, x \eta_{k}\right) .
\end{aligned}
$$

side, and the direct link are given, respectively, by

$$
\begin{aligned}
\gamma_{\mathrm{S} k} & =\frac{P_{\mathrm{S}} g_{\mathrm{S} k}}{P_{\mathrm{R}}^{\delta} g_{k k}+1}, \\
\gamma_{(\mathrm{S}, k) \rightarrow \mathrm{D}} & \approx P_{\mathrm{R}} g_{k \mathrm{D}}+P_{\mathrm{S}} g_{\mathrm{SD}} \\
\gamma_{\mathrm{SD}} & =P_{\mathrm{S}} g_{\mathrm{SD}} .
\end{aligned}
$$

The relay selection protocol is assumed to adopt an incremental relaying fashion, similar to the incremental relaying protocol variant explained in [28], where the DT is favored as long as it can by itself support the end-to-end communication. Otherwise, one of the relays assists. The reader is referred to [28] for detailed description of the protocol. Seeking the highest instantaneous SNR when opportunistic relay selection is adopted, the end-to-end SNR via the best relay is accordingly given by

$$
\gamma_{\mathrm{e} 2 \mathrm{e}}=\max \left\{\max _{k \in \mathcal{K}}\left\{\min \left\{\gamma_{\mathrm{S} k}, \gamma_{(\mathrm{S}, k) \rightarrow \mathrm{D}}\right\}\right\}, \gamma_{\mathrm{SD}}\right\} .
$$

From the link gains independence, we notice that the SNRs of all $K$ paths are conditionally independent given the direct link SNR $\gamma_{\mathrm{SD}}$. Thus, the end-to-end SNR CDF is given by

$$
F_{\gamma_{\mathrm{e} 2 \mathrm{e}}}^{\mathrm{SDF}}(x)=\int_{0}^{x}\left(F_{\gamma_{k} \mid \gamma_{\mathrm{SD}}}^{\mathrm{SDF}}(x \mid \beta)\right)^{K} f_{\gamma_{\mathrm{SD}}}(\beta) \mathrm{d} \beta,
$$

where $F_{\gamma_{k} \mid \gamma_{\mathrm{SD}}}^{\mathrm{SDF}}(x \mid \beta)$ is raised to the $K^{\text {th }}$ power due to the independent and identically distributed (i.i.d.) link gains.

The conditional CDF of the $k^{t h}$ path given the direct link gain is given, for $0 \leq \beta \leq x$, by

$$
\begin{aligned}
F_{\gamma_{k} \mid \gamma_{\mathrm{SD}}}^{\mathrm{SDF}}(x \mid \beta) & =1-\overline{F_{\gamma_{\mathrm{S} k} \mid \gamma_{\mathrm{SD}}}^{\mathrm{SDF}}}(x \mid \beta) \overline{F_{\gamma_{(\mathrm{S}, k) \rightarrow \mathrm{D}} \mid \gamma_{\mathrm{SD}}}^{\mathrm{SDF}}}(x \mid \beta) \\
& \approx 1-\overline{F_{Z}}\left(x ; \boldsymbol{p}_{1}\right) \overline{F_{X}}\left(x-\beta ; m_{\mathrm{RD}}, P_{\mathrm{R}} \theta_{\mathrm{RD}}\right),
\end{aligned}
$$

since $\gamma_{(\mathrm{S}, k) \rightarrow \mathrm{D}} \approx \gamma_{\mathrm{RD}}+\gamma_{\mathrm{SD}} \geq \gamma_{\mathrm{SD}}$. Hence,

$$
\begin{aligned}
F_{\gamma_{\mathrm{e} 2 \mathrm{e}}}^{\mathrm{SDF}}(x) & \approx \int_{0}^{x}\left(1-\overline{F_{Z}}\left(x ; \boldsymbol{p}_{1}\right) \frac{\boldsymbol{\Gamma}\left(m_{\mathrm{RD}}, \frac{x-\beta}{P_{\mathrm{R}} \theta_{\mathrm{RD}}}\right)}{\boldsymbol{\Gamma}\left(m_{\mathrm{RD}}\right)}\right)^{K} \\
& \times \frac{\beta^{m_{\mathrm{SD}}-1} e^{-\frac{\beta}{P_{\mathrm{S}} \theta_{\mathrm{SD}}}}}{\boldsymbol{\Gamma}\left(m_{\mathrm{SD}}\right)\left(P_{\mathrm{S}} \theta_{\mathrm{SD}}\right)^{m_{\mathrm{SD}}}} \mathrm{d} \beta \\
& =\sum_{k=0}^{K}\left(\begin{array}{l}
K \\
k
\end{array}\right)\left(-\overline{F_{Z}}\left(x ; \boldsymbol{p}_{1}\right)\right)^{k} \frac{1}{\boldsymbol{\Gamma}\left(m_{\mathrm{SD}}\right)\left(P_{\mathrm{S}} \theta_{\mathrm{SD}}\right)^{m_{\mathrm{SD}}}}
\end{aligned}
$$




$$
\times \underbrace{\int_{0}^{x}\left(\frac{\boldsymbol{\Gamma}\left(m_{\mathrm{RD}}, \frac{x-\beta}{P_{\mathrm{R}} \theta_{\mathrm{RD}}}\right)}{\boldsymbol{\Gamma}\left(m_{\mathrm{RD}}\right)}\right)^{k} \beta^{m_{\mathrm{SD}}-1} e^{-\frac{\beta}{P_{\mathrm{S}} \theta_{\mathrm{SD}}} \mathrm{d} \beta}}_{\hat{\mathcal{I}}_{1}}
$$

where the binomial theorem is utilized in the last step. For integer $m_{\mathrm{RD}}$, we can use the following series expansion for the upper regularized Gamma function [41, Eq. 8.352-2]:

$$
\frac{\boldsymbol{\Gamma}\left(m_{\mathrm{RD}}, \frac{x-\beta}{P_{\mathrm{R}} \theta_{\mathrm{RD}}}\right)}{\boldsymbol{\Gamma}\left(m_{\mathrm{RD}}\right)}=e^{-\frac{x-\beta}{P_{\mathrm{R}} \theta_{\mathrm{RD}}}} \sum_{m=0}^{m_{\mathrm{RD}}-1} \frac{\left(\frac{x-\beta}{P_{\mathrm{R}} \theta_{\mathrm{RD}}}\right)^{m}}{\boldsymbol{\Gamma}(m+1)} .
$$

Hence,

$$
\hat{\mathcal{I}}_{1}=e^{-\frac{k x}{P_{\mathrm{R}} \theta_{\mathrm{RD}}}} \sum_{\sum_{n=1}^{m} \sum_{\mathrm{RD}} \hat{k}_{n}=k} \hat{C}_{\left\{\hat{k}_{n}\right\}} \hat{\mathcal{I}}_{2}
$$

where



$$
\begin{aligned}
& \hat{\eta}_{k}=\left(\frac{1}{P_{\mathrm{S}} \theta_{\mathrm{SD}}}-\frac{k}{P_{\mathrm{R}} \theta_{\mathrm{RD}}}\right) \text {, } \\
& \hat{C}_{\left\{\hat{k}_{n}\right\}}=\frac{\boldsymbol{\Gamma}(k+1)\left(\frac{1}{P_{\mathrm{R}} \theta_{\mathrm{RD}}}\right)^{\sum_{n=1}^{m_{\mathrm{RD}}} \hat{k}_{n}(n-1)}}{\prod_{n=1}^{m_{\mathrm{RD}}}\left(\boldsymbol{\Gamma}\left(\hat{k}_{n}+1\right) \boldsymbol{\Gamma}(n)^{\hat{k}_{n}}\right)}, \\
& \hat{D}_{\left\{\hat{k}_{n}\right\}}=\sum_{n=1}^{m_{\mathrm{RD}}} \hat{k}_{n}(n-1),
\end{aligned}
$$

with (36) obtained via the multinomial theorem. Now, the integral $\hat{\mathcal{I}}_{2}$ in (37) is on the form of the Riemann-Liouville integral in [41, Eq. 3.383-1] when $\hat{\eta}_{k} \leq 0$. Hence,

$$
\begin{aligned}
\hat{\mathcal{I}}_{2}= & x^{\hat{D}_{\left\{\hat{k}_{n}\right\}}+m_{\mathrm{SD}}} \mathcal{B}\left(\hat{D}_{\left\{\hat{k}_{n}\right\}}+1, m_{\mathrm{SD}}\right) \\
& \times{ }_{\mathbf{1}} \boldsymbol{F}_{\mathbf{1}}\left(m_{\mathrm{SD}} ; \hat{D}_{\left\{\hat{k}_{n}\right\}}+m_{\mathrm{SD}}+1 ;-x \hat{\eta}_{k}\right) .
\end{aligned}
$$

On the other hand, when $\hat{\eta}_{k}>0$, we can use the change of variables $y=x-\beta$ and again use [41, Eq. 3.383-1] to get

$$
\begin{aligned}
\hat{\mathcal{I}}_{2}= & e^{-x \hat{\eta}_{k}} x^{\hat{D}_{\left\{\hat{k}_{n}\right\}}+m_{\mathrm{SD}}} \boldsymbol{\mathcal { B }}\left(m_{\mathrm{SD}}, \hat{D}_{\left\{\hat{k}_{n}\right\}}+1\right) \\
& \times{ }_{\mathbf{1}} \boldsymbol{F}_{\mathbf{1}}\left(\hat{D}_{\left\{\hat{k}_{n}\right\}}+1 ; \hat{D}_{\left\{\hat{k}_{n}\right\}}+m_{\mathrm{SD}}+1 ; x \hat{\eta}_{k}\right) .
\end{aligned}
$$

Substituting back, we get the end-to-end SINR CDF in (43).

\section{Selection Feasibility in Underlay Settings}

In underlay settings, an interference threshold, $I_{\mathrm{th}}$, is imposed on the secondary system. Now, we are interested in the probability to have $L$ out of the $K$ available relays that satisfy $I_{\text {th }}$. Let us denote this probability by $\mathcal{P}_{L}$. We first start with the scenarios where the direct link is not utilized.

\section{A. No Direct $\mathrm{S}-\mathrm{D}$ Link}

For the NDL setting, the end-to-end SINR CDF is given as

$$
F_{\gamma_{\mathrm{e} 2 \mathrm{e}}}^{\mathrm{NDL}}(x)=\sum_{L=0}^{K} F_{\gamma_{\mathrm{e} 2 \mathrm{e}}}^{\mathrm{NDL}}(x \mid L) \mathcal{P}_{L},
$$

where $F_{\gamma_{\mathrm{e} 2 \mathrm{e}}}^{\mathrm{NDL}}(x \mid L)$ is that derived in (16) when $L$ relays are available. The same expression applies to the IDL scenario using (24) where the direct link is not leveraged. Specifically,

$$
F_{\gamma_{\mathrm{e} 2 \mathrm{e}}}^{\mathrm{IDL}}(x)=\sum_{L=0}^{K} F_{\gamma_{\mathrm{e} 2 \mathrm{e}}}^{\mathrm{IDL}}(x \mid L) \mathcal{P}_{L}
$$

Now, we derive the probability of $L$ feasible relays, $\mathcal{P}_{L}$. When the $k^{\text {th }}$ relay is active, the interference constraint is given by (6). Since the source interference is a common random variable when any of the $K$ relays is selected, the superimposed interference signals are correlated. However, they are conditionally independent given $I_{\mathrm{SP}}$. Conditioned on $I_{\mathrm{SP}}=\beta \geq 0$, the probability that the $k^{\mathrm{th}}$ relay is feasible is given by

$F_{I_{k} \mid I_{\mathrm{SP}}}\left(I_{\mathrm{th}} \mid \beta\right)= \begin{cases}F_{X}\left(I_{\mathrm{th}}-\beta ; m_{\mathrm{RP}}, P_{\mathrm{R}} \theta_{\mathrm{RP}}\right), & \text { if } I_{\mathrm{th}}>\beta, \\ 0, & \text { elsewhere. }\end{cases}$

Accordingly, conditioned on $I_{\mathrm{SP}}=\beta$ for $0 \leq \beta \leq I_{\mathrm{th}}$, and due to the considered symmetric scenario, $\mathcal{P}_{L \mid I_{\mathrm{SP}}}(\bar{\beta})$ is given in terms of a binomial distribution as

$$
\mathcal{P}_{L \mid I_{\mathrm{SP}}}(\beta)=\left(\begin{array}{c}
K \\
L
\end{array}\right) \sum_{l=0}^{L}\left(\begin{array}{c}
L \\
l
\end{array}\right)(-1)^{l}\left(\frac{\boldsymbol{\Gamma}\left(m_{\mathrm{RP}}, \frac{I_{\mathrm{th}}-\beta}{P_{\mathrm{R}} \theta_{\mathrm{RP}}}\right)}{\boldsymbol{\Gamma}\left(m_{\mathrm{RP}}\right)}\right)^{K-L+l},
$$

where the binomial expansion is again utilized. It is straightforward to see that when $\beta>I_{\mathrm{th}}, \mathcal{P}_{L \mid I_{\mathrm{SP}}}(\beta)=0$ for $L=1,2, \cdots, K$, while $\mathcal{P}_{L \mid I_{\mathrm{SP}}}(\beta)=1$ for $L=0$. Hence, for the case when $L=0$, the support of $\beta$ is $0 \leq \beta \leq \infty$, while it is $0 \leq \beta \leq I_{\text {th }}$ for $1 \leq L \leq K$. Now, we can obtain $\mathcal{P}_{L}$ for $L=1,2, \cdots, K$ as

$$
\mathcal{P}_{L}=\left(\begin{array}{c}
K \\
L
\end{array}\right) \sum_{l=0}^{L} \frac{\left(\begin{array}{c}
L \\
l
\end{array}\right)(-1)^{l}}{\Gamma\left(m_{\mathrm{SP}}\right)\left(P_{\mathrm{S}} \theta_{\mathrm{SP}}\right)^{m_{\mathrm{SP}}}} \check{\mathcal{I}}_{1},
$$

where

$$
\check{\mathcal{I}}_{1}=\int_{0}^{I_{\mathrm{th}}}\left(\frac{\boldsymbol{\Gamma}\left(m_{\mathrm{RP}}, \frac{I_{\mathrm{th}}-\beta}{P_{\mathrm{R}} \theta_{\mathrm{RP}}}\right)}{\boldsymbol{\Gamma}\left(m_{\mathrm{RP}}\right)}\right) \beta^{K-L+l} \beta^{m_{\mathrm{SP}}-1} e^{-\frac{\beta}{P_{\mathrm{S}} \theta_{\mathrm{SP}}}} \mathrm{d} \beta .
$$

The integral in the last step can be evaluated with the aid of the multinomial theorem expansion as previously done in (34). Specifically, it is given by

$$
\check{\mathcal{I}}_{1}=e^{-\frac{(K-L+l) I_{\mathrm{th}}}{P_{\mathrm{R}}{ }^{\mathrm{RP}}}} \sum_{\sum_{n=1}^{m_{\mathrm{RP}}}}{\check{\check{C}_{n}=K-L+l}}_{\left\{\check{k}_{n}\right\}} \check{\mathcal{I}}_{2},
$$




$$
\begin{aligned}
& F_{\gamma_{\mathrm{e} 2 \mathrm{e}}}^{\mathrm{SDF}}(x) \approx \sum_{k=0}^{K}\left(\begin{array}{c}
K \\
k
\end{array}\right)\left(-\frac{\boldsymbol{\Gamma}\left(m_{\mathrm{SR}}, \frac{x}{P_{\mathrm{S}} \theta_{\mathrm{SR}}}\right)}{\boldsymbol{\Gamma}\left(m_{\mathrm{SR}}\right)}+\frac{\exp \left(-\frac{1}{2}\left(\frac{x}{P_{\mathrm{S}} \theta_{\mathrm{SR}}}-\frac{1}{P_{\mathrm{R}}^{\delta} \theta_{\mathrm{RR}}}\right)\right)}{\boldsymbol{\Gamma}\left(m_{\mathrm{SR}}\right)}\left(\frac{x}{P_{\mathrm{S}} \theta_{\mathrm{SR}}}\right)^{m_{\mathrm{SR}}}\right. \\
& \left.\times \sum_{l=0}^{m_{\mathrm{RR}}-1} \frac{\left(\frac{x}{P_{\mathrm{S}} \theta_{\mathrm{SR}}}+\frac{1}{P_{\mathrm{R}}^{\delta} \theta_{\mathrm{RR}}}\right)^{-\frac{m_{\mathrm{SR}}+l+1}{2}}}{\left(P_{\mathrm{R}}^{\delta} \theta_{\mathrm{RR}}\right)^{l}} \boldsymbol{W}_{\frac{m_{\mathrm{SR}}-l-1}{2}, \frac{-m_{\mathrm{SR}}-l}{2}}\left(\frac{x}{P_{\mathrm{S}} \theta_{\mathrm{SR}}}+\frac{1}{P_{\mathrm{R}}^{\delta} \theta_{\mathrm{RR}}}\right)\right)^{k} \\
& \times \frac{1}{\Gamma\left(m_{\mathrm{SD}}\right)\left(P_{\mathrm{S}} \theta_{\mathrm{SD}}\right)^{m_{\mathrm{SD}}}} e^{-\frac{k x}{P_{\mathrm{R}} \theta_{\mathrm{RD}}}} \sum_{\sum_{n=1}^{m} m_{\mathrm{RD}} \hat{k}_{n}=k} \hat{C}_{\left\{\hat{k}_{n}\right\}} \hat{\mathcal{I}}_{2} .
\end{aligned}
$$

where

$$
\begin{aligned}
\check{\mathcal{I}}_{2} & =\int_{0}^{I_{\mathrm{th}}}\left(I_{\mathrm{th}}-\beta\right)^{\check{D}_{\left\{\check{k}_{n}\right\}} \beta^{m_{\mathrm{SP}}-1} e^{-\beta \check{\eta}_{l}} \mathrm{~d} \beta,} \\
\check{\eta}_{l} & =\left(\frac{1}{P_{\mathrm{S}} \theta_{\mathrm{SP}}}-\frac{K-L+l}{P_{\mathrm{R}} \theta_{\mathrm{RP}}}\right), \\
\check{C}_{\left\{\check{k}_{n}\right\}} & =\frac{\boldsymbol{\Gamma}(K-L+l+1)\left(\frac{1}{P_{\mathrm{R}} \theta_{\mathrm{RP}}}\right)^{\sum_{n=1}^{m_{\mathrm{RP}} \check{k}_{n}(n-1)}}}{\prod_{n=1}^{m_{\mathrm{RP}}}\left(\boldsymbol{\Gamma}\left(\check{k}_{n}+1\right) \Gamma(n)^{\check{k}_{n}}\right)}, \\
\check{D}_{\left\{\check{k}_{n}\right\}} & =\sum_{n=1}^{m_{\mathrm{RP}}} \check{k}_{n}(n-1),
\end{aligned}
$$

with (49) obtained via the multinomial theorem [37, Section 24.1.2]. The integral $\check{\mathcal{I}}_{2}$ in (50) is on the form of the RiemannLiouville integral in [41, Eq. 3.383-1] when $\check{\eta}_{l}<0$. Hence,

$$
\begin{aligned}
\check{\mathcal{I}}_{2}= & I_{\mathrm{th}}^{\check{D}_{\left\{\check{k}_{n}\right\}}+m_{\mathrm{SP}}} \mathcal{B}\left(\check{D}_{\left\{\check{k}_{n}\right\}}+1, m_{\mathrm{SP}}\right) \\
& \times{ }_{\mathbf{1}} \boldsymbol{F}_{\mathbf{1}}\left(m_{\mathrm{SP}} ; \check{D}_{\left\{\check{k}_{n}\right\}}+m_{\mathrm{SP}}+1 ;-I_{\mathrm{th}} \check{\eta}_{l}\right) .
\end{aligned}
$$

When $\check{\eta}_{l}>0$, we can again use the change of variable $y=$ $I_{\mathrm{th}}-\beta$ and then [41, Eq. 3.383-1] to get

$$
\begin{aligned}
\check{\mathcal{I}}_{2}= & e^{-I_{\mathrm{th}} \check{\eta}_{l}} I_{\mathrm{th}}^{\check{D}_{\left\{\check{k}_{n}\right\}}+m_{\mathrm{SP}}} \mathcal{B}\left(m_{\mathrm{SP}}, \check{D}_{\left\{\check{k}_{n}\right\}}+1\right) \\
& \times{ }_{\mathbf{1}} \boldsymbol{F}_{\mathbf{1}}\left(\check{D}_{\left\{\check{k}_{n}\right\}}+1 ; \check{D}_{\left\{\check{k}_{n}\right\}}+m_{\mathrm{SP}}+1 ; I_{\mathrm{th}} \check{\eta}_{l}\right) .
\end{aligned}
$$

Therefore, $\mathcal{P}_{L}$ is finally given by (56).

For $L=0, \mathcal{P}_{0}$ is given by

$$
\begin{aligned}
\mathcal{P}_{0}= & \int_{0}^{I_{\mathrm{th}}}\left(\frac{\boldsymbol{\Gamma}\left(m_{\mathrm{RP}}, \frac{I_{\mathrm{th}}-\beta}{P_{\mathrm{R}} \theta_{\mathrm{RP}}}\right)}{\boldsymbol{\Gamma}\left(m_{\mathrm{RP}}\right)}\right)^{K} \\
& \times \frac{\beta^{m_{\mathrm{SP}}-1} e^{-\frac{\beta}{P_{\mathrm{S}} \theta_{\mathrm{SP}}}}}{\boldsymbol{\Gamma}\left(m_{\mathrm{SP}}\right)\left(P_{\mathrm{S}} \theta_{\mathrm{SP}}\right)^{m_{\mathrm{SP}}}} \mathrm{d} \beta+\frac{\boldsymbol{\Gamma}\left(m_{\mathrm{SP}}, \frac{I_{\mathrm{th}}}{P_{\mathrm{S}} \theta_{\mathrm{SP}}}\right)}{\boldsymbol{\Gamma}\left(m_{\mathrm{SP}}\right)} .
\end{aligned}
$$

Again, the integral involving higher powers of the upper regularized Gamma function is solved via its series expansion, multinomial theorem, then with the aid of the RiemannLiouville integral in [41, Eq. 3.383-1] for $\left(\frac{1}{P_{\mathrm{S}} \theta_{\mathrm{SD}}}-\frac{K}{P_{\mathrm{R}} \theta_{\mathrm{RP}}}\right)<$ 0 to yield (58) or, alternatively for $\left(\frac{1}{P_{\mathrm{S}} \theta_{\mathrm{SD}}}-\frac{K}{P_{\mathrm{R}} \theta_{\mathrm{RP}}}\right)>0$, we get (59) with

$$
\dot{D}_{\left\{\dot{k}_{n}\right\}}=\sum_{n=1}^{m_{\mathrm{RP}}} \dot{k}_{n}(n-1) .
$$

\section{B. Leveraging the Direct $\mathrm{S}-\mathrm{D}$ Link}

This case applies to the protocols which leverage the information that directly arrives at the destination from the source, i.e., in the IDL/DT and SDF protocols. The analysis of $P_{L}$ follows directly from the NDL case for $L=1,2, \cdots, K$. However, when the direct $\mathrm{S}-\mathrm{D}$ link is exploited, communication can still take place even when no feasible relays exist. Thus, the event with probability $\mathcal{P}_{0}$ in the NDL case is now further split into two sub-events. Specifically, communication can still succeed in the sub-event when the sum of the source and relay transmissions do not satisfy the interference constraint for all relays but the source alone does. Let us denote the probability of this sub-event by $\tilde{\mathcal{P}}_{0}$. It is then given by

$$
\tilde{\mathcal{P}}_{0}=\mathbb{P}\left\{\left(\bigcap_{k=1}^{K}\left(I_{k}>I_{\text {th }}\right)\right) \cap\left(I_{\text {SP }} \leq I_{\text {th }}\right)\right\} .
$$

Note that $I_{k}$ depends on $I_{\mathrm{SP}}$. Nonetheless, $\left\{I_{k}\right\}_{k=1}^{K}$ are mutually independent given $I_{\mathrm{SP}}$, and distributed as in (46).

Finally, when $I_{\mathrm{SP}}>I_{\mathrm{th}}$, with probability $\mathcal{P}_{0}-\tilde{\mathcal{P}}_{0}$, communication fails, resulting in an end-to-end SNR of $\gamma_{\mathrm{e} 2 \mathrm{e}}=0$. Hence, the distribution is a unit-step function at $\gamma_{\mathrm{e} 2 \mathrm{e}}=0$. We can now write the end-to-end SNR CDF as

$$
F_{\gamma_{\mathrm{e} 2 \mathrm{e}}}^{\mathrm{DL}}(x)=\mathcal{P}_{0}-\overline{F_{\gamma_{\mathrm{SD}}}}(x) \tilde{\mathcal{P}}_{0}+\sum_{L=1}^{K} F_{\gamma_{\mathrm{e} 2 \mathrm{e}}}^{\mathrm{DL}}(x \mid L) \mathcal{P}_{L},
$$

with $\mathrm{DL} \in\{\mathrm{IDL} / \mathrm{DT}, \mathrm{SDF}\}$ using (27) and (43). $\tilde{\mathcal{P}}_{0}$ is simply given as

$$
\tilde{\mathcal{P}}_{0}=\mathcal{P}_{0}-\frac{\boldsymbol{\Gamma}\left(m_{\mathrm{SP}}, \frac{I_{\mathrm{th}}}{P_{\mathrm{S}} \theta_{\mathrm{SP}}}\right)}{\boldsymbol{\Gamma}\left(m_{\mathrm{SP}}\right)} .
$$

\section{Diversity Analysis of Full-Duplex Relay SELECTION PROTOCOLS}

Here, we analyze the diversity order offered by each of the cooperative protocols considered in the previous sections. 






$$
\begin{aligned}
& \times I_{\mathrm{th}}^{\dot{D}_{\left\{\hat{k}_{n}\right\}}+m_{\mathrm{SP}}} \mathcal{B}\left(\dot{D}_{\left\{\hat{k}_{n}\right\}}+1, m_{\mathrm{SP}}\right) \times{ }_{\mathbf{1}} \boldsymbol{F}_{\mathbf{1}}\left(m_{\mathrm{SP}} ; \dot{D}_{\left\{\hat{k}_{n}\right\}}+m_{\mathrm{SP}}+1 ;-I_{\mathrm{th}}\left(\frac{1}{P_{\mathrm{S}} \theta_{\mathrm{SD}}}-\frac{K}{P_{\mathrm{R}} \theta_{\mathrm{RP}}}\right)\right) .
\end{aligned}
$$

$$
\begin{aligned}
& \mathcal{P}_{0}=\frac{\boldsymbol{\Gamma}\left(m_{\mathrm{SP}}, \frac{I_{\mathrm{th}}}{P_{\mathrm{S}} \theta_{\mathrm{SP}}}\right)}{\boldsymbol{\Gamma}\left(m_{\mathrm{SP}}\right)}+\frac{e^{-\frac{K I_{\mathrm{th}}}{P_{\mathrm{R}} \theta_{\mathrm{RP}}}} e^{-I_{\mathrm{th}}\left(\frac{1}{P_{\mathrm{S}} \theta_{\mathrm{SD}}}-\frac{K}{P_{\mathrm{R}} \theta_{\mathrm{RP}}}\right)}}{\boldsymbol{\Gamma}\left(m_{\mathrm{SP}}\right)\left(P_{\mathrm{S}} \theta_{\mathrm{SP}}\right)^{m_{\mathrm{SP}}}} \sum_{\sum_{n=1}^{m_{\mathrm{RP}} \dot{k}_{n}=K}} \frac{\boldsymbol{\Gamma}(K+1)}{\prod_{n=1}^{m_{\mathrm{RP}}}\left(\boldsymbol{\Gamma}\left(\dot{k}_{n}+1\right) \boldsymbol{\Gamma}(n)^{\hat{k}_{n}}\right)}\left(\frac{1}{P_{\mathrm{R}} \theta_{\mathrm{RP}}}\right)^{\dot{D}_{\left\{\dot{k}_{n}\right\}}} \\
& \times I_{\mathrm{th}}^{\dot{D}_{\left\{k_{n}\right\}}+m_{\mathrm{SP}}} \mathcal{B}\left(m_{\mathrm{SP}}, \dot{D}_{\left\{k_{n}\right\}}+1\right) \times{ }_{\mathbf{1}} \boldsymbol{F}_{\mathbf{1}}\left(\dot{D}_{\left\{\hat{k}_{n}\right\}}+1 ; \dot{D}_{\left\{\dot{k}_{n}\right\}}+m_{\mathrm{SP}}+1 ; I_{\mathrm{th}}\left(\frac{1}{P_{\mathrm{S}} \theta_{\mathrm{SD}}}-\frac{K}{P_{\mathrm{R}} \theta_{\mathrm{RP}}}\right)\right) .
\end{aligned}
$$

\section{A. $M H D F-N D L$}

By setting $P_{\mathrm{S}}=P_{\mathrm{R}}=P$, and substituting the outage threshold $\eta$ into the derived CDF expression in (16) under Rayleigh fading ( $m=1$ for all fading links), the outage probability is given by

$$
\mathcal{P}_{\text {out }}^{\mathrm{NDL}}(P)=\left(1-\frac{e^{-\frac{a}{P}}}{1+b P^{\delta-1}}\right)^{K},
$$

where $a=\eta\left(\frac{1}{\pi_{\mathrm{SR}}}+\frac{1}{\pi_{\mathrm{RD}}}\right)$ and $b=\eta \frac{\pi_{\mathrm{RR}}}{\pi_{\mathrm{SR}}}$. As $P$ increases, $e^{-\frac{a}{P}}$ approaches $1-\frac{a}{P}$. Hence,

$$
\mathcal{P}_{\text {out }}^{\mathrm{NDL}}(P) \approx\left(\frac{b P^{\delta}+a}{b P^{\delta}+P}\right)^{K} .
$$

Therefore, the diversity order is given by

$$
\mathcal{D}^{\mathrm{NDL}}=\lim _{P \rightarrow \infty} K \frac{\left(\frac{b+P^{1-\delta}}{b+a P^{-\delta}}\right)}{\log (P)}=K(1-\delta) .
$$

\section{B. $M H D F-I D L$}

By substituting $P_{\mathrm{S}}=P_{\mathrm{R}}=P$ and $x=\eta$ for Rayleigh fading in (24), we get

$\mathcal{P}_{\text {out }}^{\mathrm{IDL}}(P)=\sum_{k=0}^{K}\left(\begin{array}{c}K \\ k\end{array}\right) \frac{\left(\frac{-e^{-\frac{a}{P}}}{1+b P^{\delta-1}}\right)^{k}}{c_{k}} \approx \sum_{k=0}^{K}\left(\begin{array}{c}K \\ k\end{array}\right) \frac{\left(\frac{a P^{-1}-1}{b P^{\delta-1}-1}\right)^{k}}{c_{k}}$,

where $c_{k}=\eta k \frac{\pi_{\mathrm{SD}}}{\pi_{\mathrm{RD}}}+1$. Hence,

$$
\mathcal{D}^{\mathrm{IDL}}=\lim _{P \rightarrow \infty}-\frac{\log \left(\sum_{k=0}^{K}\left(\begin{array}{c}
K \\
k
\end{array}\right) \frac{\left(\frac{a P^{-1}-1}{b P^{\delta-1}+1}\right)^{k}}{c_{k}}\right)}{\log (P)}=0
$$

regardless of the exact value of $0 \leq \delta \leq 1$, since $\mathcal{P}_{\text {out }}^{\mathrm{IDL}}(P)$ is finite and not a function of $P$ as $P \rightarrow \infty$.

\section{C. $M H D F-I D L / D T$}

Setting $P_{\mathrm{S}}=P_{\mathrm{R}}=P$ and $x=\eta$ for Rayleigh fading in (27) yields

$$
\begin{aligned}
\mathcal{P}_{\text {out }}^{\mathrm{IDL} / \mathrm{DT}}(P) & =\sum_{k=0}^{K}\left(\begin{array}{c}
K \\
k
\end{array}\right)\left(\frac{-e^{-\frac{a}{P}}}{b P^{\delta-1}+1}\right)^{k} \frac{\left(1-e^{-\frac{d_{k}}{P}}\right)}{c_{k}} \\
& \approx \sum_{k=0}^{K}\left(\begin{array}{c}
K \\
k
\end{array}\right)\left(\frac{a-P}{b P^{\delta}+P}\right)^{k} \frac{\eta}{\pi_{\mathrm{SD}}} \frac{1}{P} \\
& =\frac{\eta}{\pi_{\mathrm{SD}}} \frac{1}{P}\left(1+\frac{a-P}{b P^{\delta}+P}\right)^{K} \\
& =\frac{\eta}{\pi_{\mathrm{SD}}} \frac{1}{P}\left(\frac{b+a P^{-\delta}}{b+P^{1-\delta}}\right)^{K},
\end{aligned}
$$

with $d_{k}=\eta\left(\frac{\eta k}{\pi_{\mathrm{RD}}}+\frac{1}{\pi_{\mathrm{SD}}}\right), \frac{d_{k}}{c_{k}}=\frac{\eta}{\pi_{\mathrm{SD}}}$, while (69) follows from the binomial theorem. Hence, the diversity gain is given by

$$
\begin{aligned}
\mathcal{D}^{\mathrm{IDL} / \mathrm{DT}} & =\lim _{P \rightarrow \infty}-\frac{\log \left(\frac{\eta}{\pi_{\mathrm{SD}}} \frac{1}{P}\left(\frac{b+a P^{-\delta}}{b+P^{1-\delta}}\right)^{K}\right)}{\log (P)} \\
& =K(1-\delta)+1 .
\end{aligned}
$$

D. $S D F$

From (43), with Rayleigh fading and $P_{\mathrm{S}}=P_{\mathrm{R}}=P$, we get $\mathcal{P}_{\text {out }}^{\mathrm{SDF}}(P) \approx \sum_{k=0}^{K}\left(\begin{array}{c}K \\ k\end{array}\right)\left(-\frac{e^{-\frac{a}{P}}}{b P^{\delta-1}+1}\right)^{k} \frac{1-e^{-\frac{p_{k}}{P}}}{q_{k}}$ 
TABLE I: Diversity order of Full-Duplex Relay Selection Protocols with $K$ Relays



where $p_{k}=x\left(\frac{1}{P_{\mathrm{S}} \pi_{\mathrm{SD}}}-\frac{k}{P_{\mathrm{R}} \pi_{\mathrm{RD}}}\right)$ and $q_{k}=1-k \frac{P_{\mathrm{S}} \pi_{\mathrm{SD}}}{P_{\mathrm{R}} \pi_{\mathrm{RD}}}$, using the same approximation for large $P$. Clearly, this yields the same diversity order of the hybrid IDL/DT protocol. That is,

$$
\mathcal{D}^{\mathrm{SDF}}=K(1-\delta)+1 \text {. }
$$

The derived diversity results are summarized in Table I.

Discussion: As clear from the diversity results in (68) and (70) for the IDL and IDL/DT, the lost diversity gain of $K(1-\delta)$ due to treating the direct S-D link as interference in the MHDF protocol is interestingly recovered. This was made possible by simply considering the direct link as a possible diversity branch, which also adds an additional diversity order to yield $K(1-\delta)+1$. This is due to the fact that, by considering DT, the direct link interference realizations are now confined only to the lower range from 0 to the outage threshold, while all higher-value realizations are utilized in direct transmission. Also, comparing (70) and (72), we find that such a hybrid MHDF-IDL/DT full-duplex relay selection protocol offers the same diversity order of the SDF protocol which jointly exploits the relayed and direct paths instead of treating one as interference. Hence, although SDF still yields better error performance, IDL/DT may still offer a close performance as the number of available relays increase, and hence, it may be favored for employment in relay selection scenarios at high SNR due to its simpler decoding approach.

\section{NUMERICAL RESULTS AND COMPARISONS}

We numerically evaluate the performance of opportunistic FDRS in underlay scenarios, and verify the theoretical findings derived in previous sections. All numerical results are evaluated by averaging over $10^{7}$ sets of channel realizations with the parameters summarized in the caption of each figure. Also, for clarity of presentation, solid lines with unfilled marks are used to plot the theoretical results, while the same filled marks with no connecting lines are used for simulation results. Hence, curves with both solid lines and filled marks indicate perfect matching between theoretical and simulation results.

We compare the studied FDRS protocols to HDRS, exploiting the direct link whenever it exists, either using maximumratio combining (MRC) or SDF protocols. The results for MRC-HDRS can be found in the literature, for instance in [42], [43], while that of SDF-HDRS, in addition to the incorporation of the primary receiver interference limit, can be easily derived using similar approaches to those in the presented manuscript.



(a) Non-cognitive setting.



(b) Cognitive setting, with $I_{\mathrm{th}}=3 \mathrm{~dB}$.

Fig. 2: Throughput vs. rate, for $\left(\pi_{\mathrm{SR}}, \pi_{\mathrm{RD}}, \pi_{\mathrm{RR}}, \pi_{\mathrm{SD}}, \pi_{\mathrm{SP}}\right.$, $\left.\pi_{\mathrm{RP}}\right)=(15,15,3,5,0,1) \mathrm{dB},\left(m_{\mathrm{SR}}, m_{\mathrm{RD}}, m_{\mathrm{RR}}, m_{\mathrm{SD}}, m_{\mathrm{SP}}\right.$, $\left.m_{\mathrm{RP}}\right)=(2,2,2,2,1,1), \delta=1, P_{\mathrm{S}}=P_{\mathrm{R}}=1$, and $K=3$.

When comparing fixed-rate half-duplex and full-duplex cooperative systems, we can either compare the outage performance or the end-to-end throughput. In outage performance comparison, the rate loss in HDR is captured via a doubled source rate. On the other hand, when throughput is compared, both fullduplex and half-duplex systems use the same source rate as that of DT, while the HDR rate loss is accounted for in the final throughput calculation via a multiplicative factor of half. The latter approach is adopted throughout this section. Specifically, for fixed-rate transmission, the end-to-end throughput, $\mathcal{T}$, is obtained simply as

$$
\mathcal{T}=R\left(1-\mathcal{P}_{\text {out }}\right) \text { bpcu, }
$$

where $R$ is the fixed source transmission rate in bits per channel use (bpcu), and $\mathcal{P}_{\text {out }}$ is the end-to-end outage probability when the source rate is equal to $R$. For half-duplex cooperation, the throughput expression is simply modified to

$$
\mathcal{T}^{\mathrm{HD}}=\frac{1}{2} R\left(1-\mathcal{P}_{\text {out }}\right) \text { bpcu. }
$$

\section{A. Throughput vs. Source Rate}

In Fig. 2, we plot the end-to-end throughput versus the source rate, for the non-cognitive and cognitive settings in Fig. 2a and Fig. 2b, respectively. As shown in Fig. 2a, FDRS offers a remarkable throughput gain compared to HDRS. It 




(a) Throughput vs. $K$.



(b) Outage probability vs. $K$.

Fig. 3: Outage vs. $K$, for $\left(\pi_{\mathrm{SR}}, \pi_{\mathrm{RD}}, \pi_{\mathrm{RR}}, \pi_{\mathrm{SD}}, \pi_{\mathrm{SP}}, \pi_{\mathrm{RP}}\right)=$ $(15,15,5,5,0,1) \mathrm{dB},\left(m_{\mathrm{SR}}, m_{\mathrm{RD}}, m_{\mathrm{RR}}, m_{\mathrm{SD}}, m_{\mathrm{SP}}, m_{\mathrm{RP}}\right)$ $=(2,2,2,2,1,1), \delta=1, P_{\mathrm{S}}=P_{\mathrm{R}}=1$, and $R=2$ bpcu.

can be noticed that HDRS starts to offer higher throughput only when the source rate considerably increases, which causes the adverse effect of the RSI to increase for FDRS while it is absent for HDRS. It can be also noticed that the hybrid IDL/DT protocol uniformly outperforms that of IDL due to exploiting the direct link, while SDF outperforms the other FDRS protocols whether in the presence or the absence of a direct link. In cognitive settings, as depicted in Fig. 2b, the throughput gain still exists relative to the HDRS protocols, although the throughput is scaled down for all protocols due to the interference constraint imposed by the primary receiver. It should be also noticed that the hybrid IDL/DT gives a very similar performance to that of SDF at lower values of the source rate. It is worth mentioning here that the throughput for all policies approaches zero as $R$ continues to grow due to the prohibitively high outage probability that is typically encountered at very high rates. Next, we investigate the relative gains by varying the number of cooperating relays.

\section{B. Throughput vs. Number of Relays}

The end-to-end performance is shown in Fig. 3 while varying $K$. In Fig. 3a, the throughput gain due to the use of FDRS is obvious for both settings where a primary receiver exists or not. The figure also emphasizes the finding that the hybrid IDL/DT may offer a similar performance to that of SDF. It can be also observed that HDRS yields higher

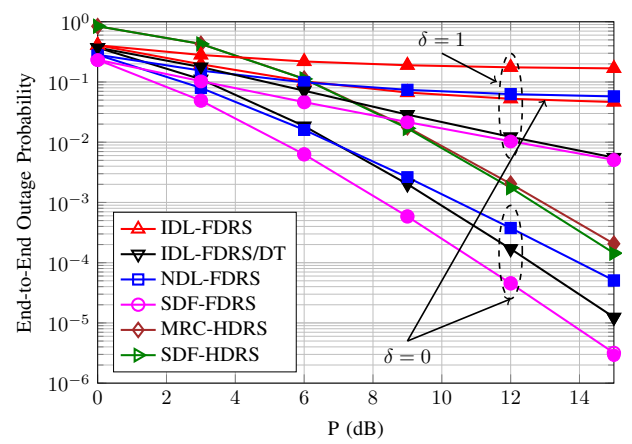

Fig. 4: Outage vs. $P$, for $\left(\pi_{\mathrm{SR}}, \pi_{\mathrm{RD}}, \pi_{\mathrm{RR}}, \pi_{\mathrm{SD}}\right)=(10,10,3$, $0) \mathrm{dB},\left(m_{\mathrm{SR}}, m_{\mathrm{RD}}, m_{\mathrm{RR}}, m_{\mathrm{SD}}\right)=(1,1,1,1), K=3$ relays, and $R=2$ bpcu.

throughput compared to FDRS in underlay settings only when the number of available relays is very limited, since the higher imposed interference by FDRS yields lower feasible relay availability, and hence, lower cooperation probability. As $K$ increases, the feasibility problem is alleviated, and the spectral efficiency gain effect in FDRS starts to take over. The choice of $I_{\mathrm{th}}=3 \mathrm{~dB}$ represents a stringent interference constraint that is only twice the noise level. As $I_{\mathrm{th}}$ increases, the throughput is enhanced to gradually approach that of the unconstrained scenario shown on the same figure. The corresponding outage performance is shown in Fig. $3 \mathrm{~b}$ for a complete demonstration of the results. By simultaneously checking the figures $3 \mathrm{a}$ and $3 \mathrm{~b}$, the reason for the throughput saturation in Fig. 3a can be noticed. Specifically, when $I_{\mathrm{th}}=3 \mathrm{~dB}$ is imposed, the outage probability saturates, causing throughput saturation. On the other hand, the non-cognitive system's throughput saturates, despite a monotonically decreasing outage probability, since the value of the source rate $(R=2 \mathrm{bpcu})$ has been already reached as shown.

\section{Outage vs. Transmit Power}

We show in Fig. 4 the outage performance of the studied protocols while varying the transmit power, $P_{\mathrm{S}}=P_{\mathrm{R}}=P$. This readily shows the offered diversity order of the cooperative protocols by comparing the slope of each. The diversity order of the HDRS clearly does not depend on the value of $\delta$, with the MRC protocol achieving a diversity of order $K=3$ while the SDF protocol attains a diversity order of $K+1=4$. As derived in the previous section, the MHDF-IDL-FDRS protocol suffers from an error floor indicating a zero diversity order regardless of the scaling trend of the RSI link with the relay's power. The MHDF-NDL-FDRS protocol performs better as it does not suffer from direct link interference, yet it still suffers from an error floor for $\delta=1$. When $\delta=0$, MHDF-NDL-FDRS achieves a diversity of order $K=3$.

For the hybrid IDL/DT protocol, the diversity order is significantly enhanced for $\delta=0$ from 0 to $K+1$ only by leveraging the direct link as a diversity branch on its own, even while still considering it as interference to the relayed paths. It is also enhanced from 0 to 1 for $\delta=1$. This result is due to the fact that the outage event caused by the direct link 
to all the relayed paths due to its large realizations is not only eliminated, but also successful communication can take place via DT. Hence, only low realizations of the direct link can now contribute to the outage of the relayed paths, limiting the denominator of the SINR for each, and recovering the diversity of each path to an order of 1 , in addition to that offered by the DT itself. When $\delta=1$, however, each path still suffers from an error floor due to the persistence if the RSI effect, and hence only a diversity of order 1 is offered due to DT. It is clear that SDF-FDRS offers the same diversity order for similar reasons, although it also leverages the direct link information even in the cooperative mode yielding a better error performance.

\section{CONCLUSION}

In this work, exact performance analysis was performed for opportunistic DF FDRS, taking the RSI of the relays into account. More specifically, the exact CDF of the endto-end SNR was derived considering Nakagami- $m$ fading channels. Moreover, the performance of FDRS techniques was analyzed in underlay networks where a primary user dictates an interference threshold that cannot be exceeded by the secondary transmissions. The performance is analyzed for both relaying settings where either coverage extension or throughput enhancement is targeted. The derived exact analytical results are shown to perfectly match with those obtained via numerical simulations. Moreover, the paper's findings confirm earlier results in AF FDRS [14] which show that an error floor exists at high SNR regimes in coverage extension scenarios where no direct link exists. Nonetheless, a diversity order of 1 can be still maintained in the existence of a direct link for simple protocols that treat the direct link as interference to the relayed paths, while a diversity of order 2 can be offered by selective FDR. These low diversity order values apply only when the RSI is linearly scaling with the relay's power. In the case of constant scaling, which agrees which some state-of-the-art adaptive suppression/cancellation techniques, a diversity order equal to the number of relays can be maintained.

\section{ACKNOWLEDGMENT}

The first author would like to thank Prof. Ahmed K. Sultan Salem at King Abdullah University of Science and Technology (KAUST) for the frequent fruitful discussions and the continued support. The first author would also like to thank Dr. Elsayed Ahmed at Qualcomm Inc. for the discussion on stateof-the-art results on the modeling of full-duplex radios.



Mohammad Galal Khafagy (S'06-M'15-SM'17) is a postdoctoral fellow at the College of Engineering, Qatar University, Doha, Qatar. He received his B.Sc. degree in Electrical Engineering from the Faculty of Engineering, Alexandria University, Egypt in 2009 $\mathrm{He}$ then joined the Wireless Intelligent Networks Center (WINC), Nile University, Egypt, from which he obtained his M.Sc. degree in Wireless Communications in 2011. He obtained his Ph.D. degree from King Abdullah University of Science and Technology (KAUST), Thuwal, Makkah Province, Saudi Arabia in 2016. His research interests include multiple-antenna techniques, buffer-aided cooperation, performance analysis and protocol design over fading channels, proactive communications, in addition to machine learning.



Mohamed-Slim Alouini (S'94-M'98-SM'03-F'09) was born in Tunis, Tunisia. He received the Ph.D. degree in Electrical Engineering from the California Institute of Technology (Caltech), Pasadena, CA, USA, in 1998. He served as a faculty member in the University of Minnesota, Minneapolis, MN, USA, then in the Texas A\&M University at Qatar, Education City, Doha, Qatar before joining King Abdullah University of Science and Technology (KAUST), Thuwal, Makkah Province, Saudi Arabia as a Professor of Electrical Engineering in 2009. His current research interests include the modeling, design, and performance analysis of wireless communication systems.



Sonia Aïssa (S'93-M'00-SM'03) received her Ph.D. degree in Electrical and Computer Engineering from McGill University, Montreal, QC, Canada, in 1998 Since then, she has been with the Institut National de la Recherche Scientifique-Energy, Materials and Telecommunications Center (INRS-EMT), University of Quebec, Montreal, QC, Canada, where she is a Full Professor.

From 1996 to 1997, she was a Researcher with the Department of Electronics and Communications of Kyoto University, and with the Wireless Systems Laboratories of NTT, Japan. From 1998 to 2000, she was a Research Associate at INRS-EMT. In 2000-2002, while she was an Assistant Professor, she was a Principal Investigator in the major program of personal and mobile communications of the Canadian Institute for Telecommunications Research, leading research in radio resource management for wireless networks. From 2004 to 2007, she was an Adjunct Professor with Concordia University, Montreal. In 2006, she was Visiting Invited Professor with the Graduate School of Informatics, Kyoto University, Japan. Her research interests lie in the area of wireless and mobile communications, and include radio resource management, cross-layer design and optimization, design and analysis of multiple antenna (MIMO) systems, cognitive and cooperative transmission techniques, performance evaluation, and energy efficiency, with a focus on Cellular and Cognitive Radio networks.

Dr. Aïssa is the Founding Chair of the IEEE Women in Engineering Affinity Group in Montreal, 2004-2007; acted as TPC Leading Chair or Cochair of the Wireless Communications Symposium at IEEE ICC in 2006, 2009, 2011 and 2012; PHY/MAC Program Cochair of the 2007 IEEE WCNC; TPC Cochair of the 2013 IEEE VTC-spring; and TPC Symposia Cochair of the 2014 IEEE Globecom. Her main editorial activities include: Editor, IEEE Transactions on Wireless Communications, 2004-2012; Associate Editor, IEEE COMmunications Magazine, 2004-2009; Technical Editor, IEEE WiRELESS COMMUNICATIONS MAGAZINE, 2006-2010; and Associate Editor, Wiley Security and Communication Networks Journal, 2007-2012. She currently serves as Area Editor for the IEEE TRANSACTIONS ON WIRELESS COMMUNICATIONS, and Technical Editor for the IEEE COMMUNICATIONS MAGAZINE. Awards to her credit include the NSERC University Faculty Award in 1999; the Quebec Government FQRNT Strategic Faculty Fellowship in 2001-2006; the INRS-EMT Performance Award multiple times since 2004, for outstanding achievements in research, teaching and service; and the Technical Community Service Award from the FQRNT Centre for Advanced Systems and Technologies in Communications in 2007. She is co-recipient of five IEEE Best Paper Awards and of the 2012 IEICE Best Paper Award; and recipient of NSERC Discovery Accelerator Supplement Award. She is a Distinguished Lecturer of the IEEE Communications Society (ComSoc) and an Elected Member of the ComSoc Board of Governors. 


\section{REFERENCES}

[1] J. Mitola and G. Q. Maguire, Jr, "Cognitive radio: Making software radios more personal," IEEE Personal Commun. Mag., vol. 6, no. 4 pp. 13-18, Aug. 1999.

[2] A. Goldsmith, S. A. Jafar, I. Maric, and S. Srinivasa, "Breaking spectrum gridlock with cognitive radios: An information theoretic perspective," Proc. IEEE, vol. 97, no. 5, pp. 894-914, May 2009.

[3] K. Letaief and W. Zhang, "Cooperative communications for cognitive radio networks," Proc. IEEE, vol. 97, no. 5, pp. 878-893, May 2009.

[4] A. Bletsas, A. Khisti, D.P. Reed, and A. Lippman, "A simple cooperative diversity method based on network path selection," IEEE J. Sel. Areas Commun., vol. 24, no. 3, pp. 659-672, Mar. 2006.

[5] D. S. Michalopoulos and G. K. Karagiannidis, "Performance analysis of single relay selection in Rayleigh fading," IEEE Trans. Wireless Commun., vol. 7, no. 10, pp. 3718-3724, Oct. 2008.

[6] S. I. Hussain, M. M. Abdallah, M.-S. Alouini, M. Hasna, and K. Qaraqe, "Best relay selection using SNR and interference quotient for underlay cognitive networks," in Proc. IEEE ICC'12, Ottawa, Canada, June 2012.

[7] G. Chen, Y. Gong, and J. Chambers, "Study of relay selection in a multi-cell cognitive network," IEEE Wireless Commun. Lett., vol. 2, no. 4, pp. 435-438, Aug. 2013.

[8] J. Lee, H. Wang, J. G. Andrews, and D. Hong, "Outage probability of cognitive relay networks with interference constraints," IEEE Trans. Wireless Commun., vol. 10, no. 2, pp. 390-395, Feb. 2011

[9] S. Sagong, J. Lee, and D. Hong, "Capacity of reactive DF scheme in cognitive relay networks," IEEE Trans. Wireless Commun., vol. 10, no. 10, pp. 3133-3138, Oct. 2011.

[10] M. Jain, J. Choi, T. Kim, D. Bharadia, S. Seth, K. Srinivasan, P. Levis, S. Katti, and P. Sinha, "Practical, real-time, full duplex wireless," in Proc. ACM MobiCom'11, Las Vegas, NV, Sept. 2011.

[11] M. Duarte, C. Dick, and A. Sabharwal, "Experiment-driven characterization of full-duplex wireless systems," IEEE Trans. Wireless Commun. vol. 11, no. 12, pp. 4296-4307, Dec. 2012.

[12] G. Zheng, I. Krikidis, and B. Ottersten, "Full-duplex cooperative cognitive radio with transmit imperfections," IEEE Trans. Wireless Commun., vol. 12, no. 5, pp. 2498-2511, May 2013.

[13] H. Kim, S. Lim, H. Wang, and D. Hong, "Optimal power allocation and outage analysis for cognitive full duplex relay systems," IEEE Trans. Wireless Commun., vol. 11, no. 10, pp. 3754-3765, Oct. 2012

[14] I. Krikidis, H.A. Suraweera, P. J. Smith, and C. Yuen, "Full-duplex relay selection for amplify-and-forward cooperative networks," IEEE Trans. Wireless Commun., vol. 11, no. 12, pp. 4381-4393, Dec. 2012.

[15] I. Krikidis, H. A. Suraweera, S. Yang, and K. Berberidis, "Full-duplex relaying over block fading channel: A diversity perspective," IEEE Trans. Wireless Commun., vol. 11, no. 12, pp. 4524-4535, Dec. 2012.

[16] X. Rui, J. Hou, and L. Zhou, "On the performance of full-duplex relaying with relay selection," Electronics Letters, vol. 46, no. 25, pp. 1674-1676, Dec. 2010.

[17] B. Zhong, D. Zhang, Z. Zhang, Z. Pan, K. Long, and A. V. Vasilakos, "Opportunistic full-duplex relay selection for decode-and-forward cooperative networks over Rayleigh fading channels," in Proc. IEEE ICC'14, June 2014.

[18] M. G. Khafagy, M. S. Alouini, and S. Aïssa, "Full-duplex opportunistic relay selection in future spectrum-sharing networks," in Proc. IEEE ICC Wkshps'15, June 2015, pp. 1196-1200.

[19] M. G. Khafagy, M. S. Alouini, and S. Aïssa, "On the performance of future full-duplex relay selection networks," in Proc. IEEE CAMAD'15, Sept. 2015, pp. 11-16.

[20] Z. Zhang, X. Chai, K. Long, A. V. Vasilakos, and L. Hanzo, "Full duplex techniques for $5 \mathrm{~g}$ networks: self-interference cancellation, protocol design, and relay selection," IEEE Commun. Mag., vol. 53, no. 5, pp. 128-137, May 2015.
[21] B. Zhong, Z. Zhang, X. Chai, Z. Pan, K. Long, and H. Cao, "Performance analysis for opportunistic full-duplex relay selection in underlay cognitive networks," IEEE Trans. Veh. Technol., vol. 64, no. 10, pp. 4905-4910, Oct. 2015.

[22] Y. Wang, Y. Xu, N. Li, W. Xie, K. Xu, and X. Xia, "Relay selection of full-duplex decode-and-forward relaying over Nakagami- $m$ fading channels," IET Communications, vol. 10, no. 2, pp. 170-179, Feb. 2016.

[23] C. Yin, T. X. Doan, N. P. Nguyen, T. Mai, and L. D. Nguyen, "Outage probability of full-duplex cognitive relay networks with partial relay selection," in Proc. SigTelCom'17, Jan. 2017, pp. 115-118.

[24] Q. Li, S. Feng, X. Ge, G. Mao, and L. Hanzo, "On the performance of full-duplex multi-relay channels with DF relays," IEEE Trans. Veh. Technol., vol. 66, no. 10, pp. 9550-9554, Oct. 2017.

[25] ITU-R WP5D, Recommendation ITU-R M.2083, "IMT vision framework and overall objectives of the future development of IMT for 2020 and beyond," Sept. 2015.

[26] ITU-R WP5D, Report ITU-R M.2320-0, "Future technology trends of terrestrial IMT systems," Nov. 2014.

[27] M. Khafagy, A. Ismail, M. S. Alouini, and S. Aissa, "On the outage performance of full-duplex selective decode-and-forward relaying," IEEE Commun. Lett., vol. 17, no. 6, pp. 1180-1183, June 2013.

[28] M. G. Khafagy, A. Ismail, M.-S. Alouini, and S. Aissa, "Efficient cooperative protocols for full-duplex relaying over Nakagami-m fading channels," IEEE Trans. Wireless Commun., vol. 14, no. 6, pp. 34563470, June 2015.

[29] M. Amjad, F. Akhtar, M. H. Rehmani, M. Reisslein, and T. Umer, "Fullduplex communication in cognitive radio networks: A survey," IEEE Commun. Surveys Tuts., vol. PP, no. 99, pp. 1-1, 2017.

[30] E. Ahmed, A.M. Eltawil, and A. Sabharwal, "Rate gain region and design tradeoffs for full-duplex wireless communications," IEEE Trans. Wireless Commun., vol. 12, no. 7, pp. 3556-3565, July 2013.

[31] D. Bharadia, E. McMilin, and S. Katti, "Full duplex radios," in Proc. ACM SIGCOMM '13, New York, NY, USA, Aug. 2013.

[32] E. Ahmed and A.M. Eltawil, "All-digital self-interference cancellation technique for full-duplex systems," IEEE Trans. Wireless Commun. vol. 14, no. 7, pp. 3519-3532, July 2015.

[33] E. Ahmed, A. Eltawil, Z. Li, and B. Cetiner, "Full-duplex systems using multi-reconfigurable antennas," IEEE Trans. Wireless Commun., vol. 14, no. 11, pp. 5971-5983, Nov. 2015.

[34] L. Jimenez Rodriguez, N. H. Tran, and T. Le-Ngoc, "Performance of full-duplex AF relaying in the presence of residual self-interference," IEEE J. Sel. Areas Commun., vol. 32, no. 9, pp. 1752-1764, Sept. 2014.

[35] T. Kwon, S. Lim, S. Choi, and D. Hong, "Optimal duplex mode for DF relay in terms of the outage probability," IEEE Trans. Veh. Technol., vol. 59, no. 7, pp. $3628-3634$, Sept. 2010.

[36] T. Riihonen, S. Werner, and R. Wichman, "Hybrid full-duplex/halfduplex relaying with transmit power adaptation," IEEE Trans. Wireless Commun., vol. 10, no. 9, pp. 3074-3085, Sept. 2011.

[37] M. Abramowitz and I. A. Stegun, Eds., Handbook of Mathematical Functions, $10^{\text {th }}$ Printing, Dover Publications, Dec. 1972.

[38] H. Alves, G. Fraidenraich, R. D. Souza, M. Bennis, and M. Latva-aho, "Performance analysis of full duplex and selective and incremental half duplex relaying schemes," in Proc. IEEE WCNC'12, Paris, France, Apr. 2012.

[39] P. K. Sharma and P. Garg, "Outage analysis of full duplex decode and forward relaying over Nakagami- $m$ channels," in Proc. NCC'13, New Delhi, India, Feb. 2013.

[40] H. Alves, D.B. da Costa, R.D. Souza, and M. Latva-aho, "Performance of block-Markov full duplex relaying with self interference in Nakagami-m fading," IEEE Wireless Commun. Lett., vol. 2, no. 3, pp. 311-314, June 2013.

[41] I. S. Gradshteyn and I. M. Ryzhik, Table of Integrals, Series, and Products, Seventh Edition, Academic Press, 2007. 
[42] K. Yan, J. Jiang, Y. G. Wang, and H. T. Liu, "Outage probability of selection cooperation with mrc in nakagami- $m$ fading channels," IEEE Signal Process. Lett., vol. 16, no. 12, pp. 1031-1034, Dec. 2009.

[43] G. C. Alexandropoulos, A. Papadogiannis, and K. Berberidis, "Per- formance analysis of cooperative networks with relay selection over nakagami- $m$ fading channels," IEEE Signal Process. Lett., vol. 17, no. 5, pp. 441-444, May 2010. 